\title{
Folgen der COVID-19-Krise auf die kurz-, mittel- und langfristigen Beschäftigungs- und Entlohnungschancen nach Berufen
}

\author{
Tobias Maier (D) • Anke Mönnig • Gerd Zika
}

Eingegangen: 14. Januar 2020 / Angenommen: 21. Mai 2021 / Online publiziert: 21. Juni 2021

(C) Der/die Autor(en) 2021

Zusammenfassung Dieser Beitrag stellt das Modellsystem QINFORGE vor. Es ist durch die Berücksichtigung beruflicher Mobilitätsprozesse in der Lage, Anpassungsreaktionen zwischen Arbeitsangebot und -bedarf auf beruflicher Ebene in der kurzen und langen Frist aufzuzeigen. Das Modell wird genutzt, um die Auswirkungen der COVID-19-Krise auf die Beschäftigungs- und Entlohnungschancen in Berufen zu verdeutlichen. Hierfür werden zwei Szenarien miteinander vergleichen: Ein „kontrafaktisches Szenario“ schreibt langfristige Verhaltensweisen und Trends ausgehend vom Jahr 2019 fort. Die „Basisprojektion“ versucht hingegen, die Konjunktur in der kurzen Frist auf Branchenebene mit abzubilden. Hierdurch werden modellendogen Verhaltensänderungen auf Berufsebene angeregt.

Die Krise wird die Wirtschaftsleistung Deutschlands langfristig um drei Jahre zurückwerfen. Da sich aufgrund der schlechteren Arbeitsmarktlage aber auch einige Erwerbspersonen, insbesondere Frauen, vom Arbeitsmarkt zurückziehen werden, steigt die prognostizierte Zahl der Erwerbslosen in den Jahren 2020 und 2021 in der Basisprojektion nur um rund 400.000 Personen im Vergleich zum kontrafaktischen Szenario an. Langfristig stellt das knappe Arbeitsangebot hingegen einen hemmenden Faktor für das Wirtschaftswachstum dar. Zudem wird durch die Krise in der kurzen und mittleren Frist das gesamtwirtschaftliche Lohnwachstum gehemmt.

\footnotetext{
Tobias Maier $(\bowtie)$

Bundesinstitut für Berufsbildung (BIBB), Bonn, Deutschland

E-Mail: tobias.maier@bibb.de

Anke Mönnig

Gesellschaft für Wirtschaftliche Strukturforschung mbH (GWS), Osnabrück, Deutschland

E-Mail: moennig@gws-os.com
}

Gerd Zika

Institut für Arbeitsmarkt- und Berufsforschung (IAB) der Bundesagentur für Arbeit (BA), Nürnberg,

Deutschland

E-Mail: gerd.zika@iab.de 
Die Ergebnisse des Modells zeigen, dass durch die Krise vor allem Berufe in der Gastronomie, dem Tourismus, sowie in Kunst und Kultur betroffen sind. Ab dem Jahr 2025 wird hier jedoch wieder das Lohn- und Beschäftigungsniveau erreicht, das auch im kontrafaktischen Szenario erreicht worden wäre. Bei den kurzfristigen Lohnsteigerungen in den Gesundheitsberufen handelt es sich hingegen um Vorzieheffekte. Auf lange Sicht ist in der Basisprojektion gegenüber dem „,kontrafaktischen Szenario“ kein höheres Lohnniveau zu erwarten. Das Arbeitsangebot in den Gesundheitsberufen - insbesondere in der Pflege - könnte durch die Krise zwar zunehmen, dies wäre jedoch darauf zurückzuführen, dass die Erwerbschancen und Entlohnungsmöglichkeiten außerhalb der Pflegeberufe durch die COVID-19Pandemie vergleichsweise geringer werden könnten.

Schlüsselwörter Projektion · Berufsstruktur · Lohnentwicklung · COVID-19Pandemie $\cdot$ Szenarioanalyse $\cdot$ Input-Output $\cdot$ Arbeitsmarkt

\title{
Consequences of the COVID-19 crisis on short-, medium- and long- term employment and remuneration opportunities by occupations
}

\begin{abstract}
This paper presents the model system QINFORGE. By taking into account occupational mobility matrices, the model is able to show adaptation reactions between labour supply and demand at the occupational level in the short- and longterm. We use the model to illustrate the effects of the COVID-19 crisis on employment and remuneration opportunities in occupations. For this purpose, we compare two scenarios. On the one hand, a "counterfactual scenario" extrapolates long-term behaviour and trends starting from the year 2019. The "baseline projection", on the other hand, models the economic situation in the short term at the industry level. This stimulates model-based behavioural changes at the occupational level.

The crisis will set back Germany's economic performance by three years in the long-term. As the worsening labour market situation will also cause lower labour participation rates, especially among women, the number of the projected unemployed in the "baseline projection" for 2020 and 2021 will only rise by around 400,000 compared with the "counterfactual scenario". In the long term, however, the scarce supply of labour will be an inhibiting factor for economic growth. In addition, the crisis will inhibit overall wage growth in the short and medium term

The results of the model show that occupations in gastronomy, tourism, and art and culture are particularly affected by the crisis. From 2025, however, the wage and employment levels that would have been achieved in the "counterfactual scenario" will be reached. By contrast, the short-term wage increases in the health care occupations are anticipatory effects. In the long-term, the baseline-projection does not forecast higher wage levels than the "counterfactual scenario". Nevertheless, the labour supply in the health care occupations, especially in nursing, could increase in the long term because of the crisis. However, this will be because the COVID 19 pandemic may reduce the employment and wage opportunities that are available to health care professionals outside of the health care sector.
\end{abstract}


Keywords Projection - Occupational structure - Wage development - COVID-19 pandemic $\cdot$ Scenario analysis $\cdot$ Input-output $\cdot$ Labour market

\section{Einleitung}

Für die Bildungs- und Arbeitsmarktpolitik ist es relevant zu wissen, welche Art von Tätigkeiten für die Herstellung von Gütern und Erbringung von Dienstleistungen auf dem Arbeitsmarkt nachgefragt werden und inwieweit die dem Arbeitsmarkt zur Verfügung stehenden Arbeitskräfte diese Tätigkeiten ausüben können. Denn Passungsprobleme, sowohl auf beruflicher als auch auf regionaler Ebene, verursachen in der Regel volkswirtschaftliche Kosten, da Arbeitslose beispielsweise Anspruch auf Sozial- und zum Teil auch auf Versicherungsleistungen haben oder Wertschöpfung verloren geht. Für Individuen haben mögliche Erwerbs- und Entlohnungschancen hingegen unmittelbare und langfristige Konsequenzen auf ihre finanziellen Ressourcen. Wirtschaftliche Krisen, wie durch die COVID-19(Coronavirus SARS-CoV-2)Pandemie ausgelöst, können diese Erwerbs- und Entlohnungschancen gefährden. Es bedarf deshalb der Analyse, wie die COVID-19-Pandemie auf berufsspezifische Arbeitsmärkte und Entlohnungschancen einwirken könnte. Ein Blick auf den Arbeitsmarkt der Zukunft kann helfen, mögliche Problemfelder bereits frühzeitig zu erkennen und entsprechende Handlungsalternativen zu erarbeiten. Obwohl somit ein begründetes Interesse am Arbeitsmarkt der Zukunft besteht, werden praktische Annäherungsversuche, diesen zu beschreiben, kritisch betrachtet.

Die Kritik an Projektionen wird zumeist über ihre Praxisbewährung formuliert. Schließlich sei eine Vielzahl vergangener Prognosen bislang nicht eingetroffen bzw. die mit ihnen verbundenen Erwartungen nicht bestätigt. Dieser Erwartungsenttäuschung geht das Verständnis voraus, dass eine Prognose unter vollständiger Kenntnis der Entwicklung aller für die Prognose relevanten Parametern zustande kommt (Lucas 1976). Tatsächlich sind längerfristige Prognosen - egal für welche Fragestellung - jedoch immer bedingte Prognosen, deren Geltungsbereich im Sinne eines Wenn-Dann-Charakters nur innerhalb definierter Rahmenbedingungen gilt (Helmrich und Zika 2019).

Zur Lösung der Unsicherheitsthematik hat sich bei Projektionen deshalb die Szenarienanalyse (Helmrich et al. 2013) etabliert. Bei einer transparenten Nennung der Prognoseannahmen werden einzelne Parameter der Prognose verändert, um deren langfristige Auswirkungen auf weitere Größen sichtbar zu machen. Zudem ist es ratsam, die Berechnungen regelmäßig zu erneuern. Das etwaige Fortbestehen von vormals identifizierten Verhaltensweisen muss regelmäßig überprüft sowie mögliche Auswirkungen kurzfristiger Ereignisse quantifiziert werden. Die derzeitige COVID19-Pandemie stellt beispielsweise ein solch einschneidendes Ereignis dar, dessen kurz-, mittel- und langfristigen Auswirkungen auf den Arbeitsmarkt derzeit unbekannt sind.

Mit diesem Beitrag wird das Modellsystem QINFORGE vorgestellt. Es stellt den makroökonometrischen Kern der Qualifikations- und Berufsprojektionen (QuBeProjekt) dar. Es lässt eine Abschätzung der möglichen mittel- und langfristigen Auswirkungen der COVID-19-Krise zu, indem die Krise selbst als eine Art ,externer 
Schock" interpretiert wird. So lassen sich über Veränderungen in der kurzen Frist auch persistente Folgen für die Erwerbs- und Einkommenschancen nach Berufen nachzeichnen. Abschn. 2 erläutert zunächst das Modellsystem. Um die kurz-, mittel- und langfristigen Auswirkungen der COVID-19-Krise darzustellen, wird eine „Basisprojektion“, welche die derzeitig bekannten Einschnitte in der wirtschaftlichen Entwicklung abbildet, mit einem „kontrafaktischen Szenario“ verglichen. Das kontrafaktische Szenario simuliert ausgehend vom Jahr 2019 eine Welt ohne Corona-Pandemie (Abschn. 3). Abschn. 4 zeigt die Unterschiede in den Entlohnungsund Erwerbschancen nach Beruf, die sich in beiden Szenarien ergeben. Der Beitrag schließt in Abschn. 5 mit einem Fazit.

\section{Das Modellsystem QINFORGE}

Die Langfristprojektionen des QuBe-Projektes bestehen aus einer Bevölkerungsprojektion (Fuchs et al. 2017), einem darauf aufsetzenden Bildungsmodell (Kalinowski et al. 2021) und dem makroökonometrischen Input-Output-Modell INFORGE (Interindustry Forecasting Germany) (Ahlert et al. 2009; Maier et al. 2015). Alle drei Modelltypen gehen in das Modellsystem mit Namen QINFORGE ein. Die Module sind miteinander verknüpft, indem sie entweder exogenen Input für die Bestimmung von Teilgrößen in anderen Modulen liefern (wie beispielsweise die Bevölkerungsprojektion an die Bestimmung der Arbeitsnachfrage oder Arbeitsangebot) oder endogene Rückkopplungseffekte auf die Einzelmodule ausüben (wie beispielsweise gleichzeitig der Lohn das Arbeitsangebot bestimmt und das Arbeitsangebot auch die Lohnentwicklung beeinflusst). Die aus QINFORGE entstehenden Projektionen reichen bis in das Jahr 2040 und wurden bislang vorwiegend für die Untersuchung unterschiedlicher Fragestellungen zum Strukturwandel herangezogen (Mönnig et al. 2019, 2020, 2018; Zika et al. 2017, 2019).

Das Modellsystem QINFORGE hebt sich von den internationalen und nationalen Projektionsmodellen (Bakens et al. 2018; Bonin et al. 2007; Bundesamt für Statistik 2019; Bureau of Labor Statistics 2018; Cedefop 2018; France Stratégie und Dares 2018; Statistics Sweden 2017; vbw 2019; Wilson et al. 2016) ab, weil es die Kopplungsfunktion des Berufs zwischen der Arbeitsangebots- und -nachfrageseite explizit in das Zentrum der Analyse stellt. Dies ist möglich, da der erlernte Beruf der Befragten im Mikrozensus über den höchsten beruflichen Abschluss im Zusammenhang mit der Hauptfachrichtung in die nationale Klassifikation der Berufe 2010 (KldB 2010) überführt wurde (Maier und Helmrich 2012). Hierdurch können berufliche Mobilitätmatrizen zwischen erlerntem und ausgeübtem Beruf ausgewiesen und für die Gegenüberstellung des berufsspezifischen Arbeitsangebots mit dem Bedarf an Erwerbstätigen im Beruf genutzt werden. Dies ermöglicht die Identifikation von Arbeitskräfteknappheiten auf Berufsebene, welche wiederum Rückwirkungen auf die Bereitstellung von Waren und Dienstleistungen auf Branchenebene zeigen.

Der folgende Abschnitt zeigt zunächst die modulare Struktur von QINFORGE auf, um anschließend die einzelnen, für die vorliegende Fragestellung relevanten Modellelemente $\mathrm{zu}$ beschreiben. 


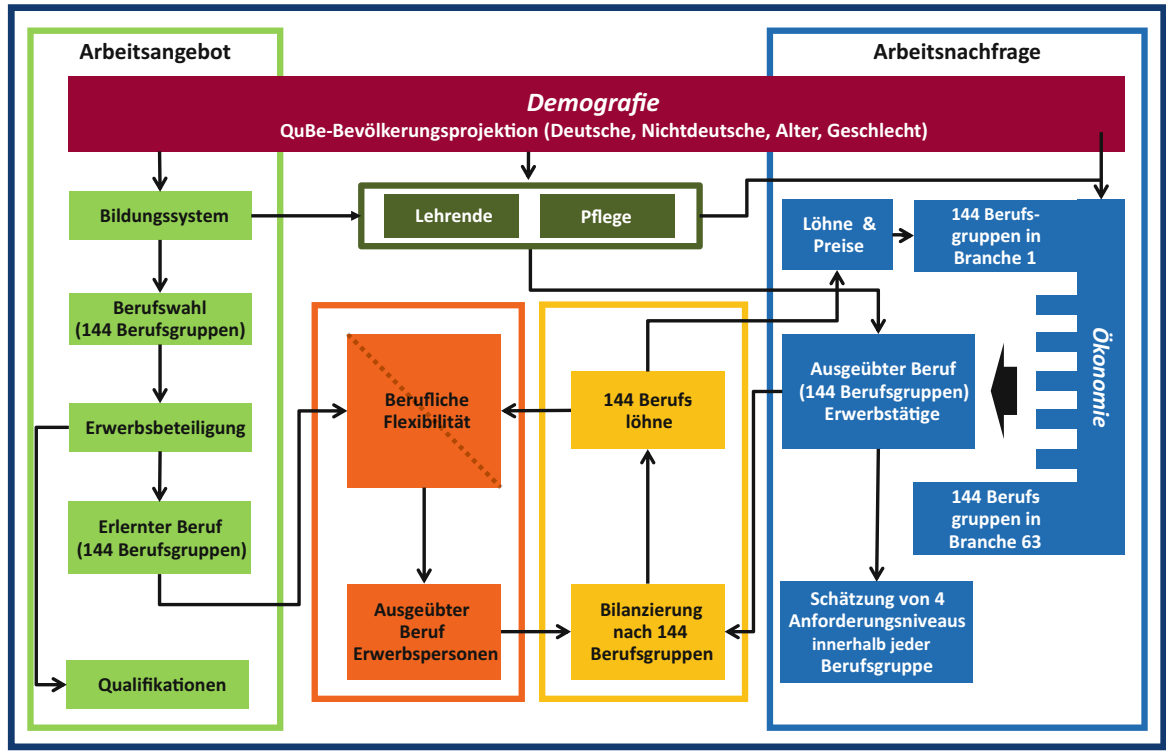

Abb. 1 Die Module des Rechenmodells QINFORGE. (Quelle: QuBe-Projekt, sechste Welle; eigene Darstellung)

\subsection{Modulare Struktur QINFORGE}

Abb. 1 gibt einen Überblick über die modulare Struktur von QINFORGE. Die demografische Entwicklung nach Alter, Geschlecht und Nationalität (roter Kasten in Abb. 1) beeinflusst sowohl das Arbeitsangebot als auch die Arbeitsnachfrage. Die Arbeitsnachfrage wird von der Bevölkerung über die Wirtschaftsentwicklung beeinflusst, wobei insbesondere die private Konsumnachfrage eine entscheidende Rolle spielt. Diese wird durch die Bevölkerung sowohl in ihrer Entwicklung (Sonnenburg et al. 2015) als auch in ihrer Struktur (Stöver und Wolter 2015; AignerWalder und Döring 2014) verändert. Darüber hinaus gehen mit einem sinkenden Erwerbspersonenpotenzial - ceteris paribus - die Produktionsmöglichkeiten in einer Volkswirtschaft zurück (Deutsche Bundesbank 2017). Da vor allem Pflege- und Erziehungsleistungen aufgrund ihrer Nachfrager eng mit der Bevölkerungsentwicklung verknüpft sind, wird die Bestimmung der Arbeitsnachfrage für diese beiden Wirtschaftszweige aus dem ökonomischen Modellkontext von INFORGE ausgegliedert und in zwei Sub-Module ausgelagert. Das Angebot an Fachkräften mit einem erlernten Beruf bestimmt sich aus der alters-, geschlechts- und nationalitätsspezifischen Berufswahl und der damit in Verbindung stehenden Erwerbsneigung (grüner Kasten in Abb. 1). Diese Erwerbsneigung wird von konjunkturellen Entwicklungen beeinflusst (Cedefop 2010; Kriechel et al. 2013).

Das Angebot an Personen nach erlerntem Beruf wird über im Mikrozensus ermittelte berufliche Flexibilitätsmatrizen in Erwerbspersonen für einen Beruf umgerechnet (oranger Kasten). Das makroökonometrische Input-Output-Modell INFORGE berechnet hierbei die Arbeitsnachfrage (blauer Kasten). Die Erwerbstätigen nach 
Beruf werden den berufsspezifischen Erwerbspersonen gegenübergestellt. Bei berufsspezifischen Knappheiten ergeben sich Veränderungen in der berufsspezifischen Lohnstruktur (gelber Kasten).

\subsection{Das Regressionsmodell INFORGE}

Die Wahl des ,richtigen“ Modelltyps hängt von der Fragestellung ab. Für eine langfristige Arbeitsmarktprojektion mit dem Ziel einer zukünftigen Engpassanalyse auf Berufsebene ist ein dynamisches Modell mit Strukturelementen notwendig. Allein diese beiden Bedingungen schließen Partialmodelle (Peichl et al. 2010) aus. Auch ausgeschlossen sind jegliche statischen Modelltypen. Ebenso unvereinbar ist damit die Philosophie des Gleichgewichtsgedankens (Meagher et al. 2015), da gerade zukünftige Ungleichgewichte aufgedeckt werden sollen. Der Input-Output-Ansatz ist für den Strukturaspekt von entscheidendem Vorteil. Das makroökonometrische Input-Output-Modell INFORGE entspricht diesen Anforderungen.

INFORGE (Ahlert et al. 2009; Maier et al. 2015) beschreibt das ökonomische Verhalten unterschiedlicher Wirtschaftsakteure ${ }^{1}$. Interindustrielle Verflechtungen werden explizit behandelt, so wie auch die Veränderung über die Zeit. Die Konten der Volkswirtschaftlichen Gesamtrechnungen (VGR) sind sowohl von der Nachfrage- als auch von der Angebotsseite ausgeglichen. Die Wirtschaftsentwicklung wird „bottom-up“ auf Wirtschaftszweig und Güterebene beschrieben und das Bruttoinlandsprodukt bestimmt sich aus der Summe seiner Teile. Die dafür notwendige hohe Anzahl an Gleichungen wird simultan gelöst. Die Input-Output-Tabellen sind dabei vollständig in das Kontensystem der VGR implementiert. Die Einkommensentstehung, -verteilung und -verwendung ist ganzheitlich abgebildet.

Bei Input-Output-Modellen werden auf der Arbeitsnachfrageseite zur Bestimmung der Erwerbstätigen in den Branchen unter anderem multi-sektorale makroökonomische Modelle verwendet, die um eine Input-Output-Tabelle aufgebaut sind (Wilson und Lindley 2007; für Deutschland: Maier et al. 2015; Prognos 2013). ${ }^{2}$ Input-Output-Tabellen beschreiben in einer sehr komprimierten Form die ökonomische Verflechtung zwischen Produktionsbereichen. Dynamische Input-Output-Modelle (Lehr et al. 2014) nutzen eine limitationale Produktionsfunktion, mit der die Produktion $y$ über die Endnachfrage $x$ und den technischen Koeffizienten $(I-A)^{-1}$ bestimmt wird.

$$
y=(I-A)^{-1} x
$$

Dynamische Input-Output-Modelle wie INFORGE kombinieren das Instrument der Ökonometrie mit der auf Input-Output-Tabellen basierenden limitationalen Pro-

\footnotetext{
1 Grundsätzlich können Sprachregeln eine diskriminierende Wirkung haben. Aus Gründen der Texteffizienz und des Leseflusses wird im Folgenden jedoch das generische Maskulinum verwendet. Selbstverständlich sind damit immer alle Geschlechter gemeint.

2 Input-Output-Modelle bilden als Totalmodelle im Gegensatz zu Partialmodellen, zu denen meist Mikromodelle (Peichl et al. 2010) gezählt werden, die gesamte Volkwirtschaft und nicht nur einen Teil davon ab.
} 
duktionsfunktion, um Anpassungsprozesse in der Wirtschaft abzubilden. Sie ähneln den dynamischen „computable general equilibrium“ (CGE)-Modellen (Meagher et al. 2015). Beide Modelltypen lösen zwar simultan und zeigen Pfadabhängigkeiten auf - außerdem fußen sie auf derselben Datenbasis und nicht-linearen Zielfunktionen - allerdings unterscheiden sie sich in der theoretischen Fundierung der Modelltypen. Makroökonometrische Input-Output-Modelle basieren in der Regel auf postkeynesianischen Eigenschaften, wie beispielsweise unfreiwillige Arbeitslosigkeit, Unsicherheit oder träge Preisbildung. Diese Modelltypen können nicht optimieren anders als die CGE-Modelle, welche auf Gleichgewichtspositionen ausgerichtet sind (West 2006). Optimierungsmöglichkeiten können hierbei nur durch Simulationsrechnungen erzeugt werden (Pollitt et al. 2019).

Der mit dem Modell INFORGE berechnete Wachstumspfad beinhaltet den technologischen Wandel - allerdings nur insoweit, als er in der Vergangenheit empirisch beobachtbar war. Hierbei definieren die technischen Koeffizienten der Input-OutputBeziehungen den technologischen Wandel (Mönnig 2016). Inputkoeffizienten sind der Anteil des Vorleistungseinsatzes eines Produkts im Verhältnis zur Produktion eines anderen Produkts. Veränderte Inputstrukturen können als Veränderungen im Produktmix innerhalb einer bestimmten Produktgruppe interpretiert werden (Meyer et al. 1999; Richter 1991). Ein autonomer technischer Fortschritt (Klauder 1986) besteht außerdem darin, dass Änderungen der Inputkoeffizienten in INFORGE endogen mit einem Zeittrend geschätzt werden.

Während die Produktion neben den technischen Koeffizienten von der heimischen Endnachfrage bestimmt wird (siehe Gl. 1), hängt die Nachfrage unter anderem von Preisveränderungen ab. Diese wiederum sind das Resultat eines Stückkostenansatzes in den jeweiligen Produktionsbereichen. Damit sind sowohl nachfrage- als auch angebotsseitige Entwicklungen in der Bestimmung der Produktion eingebunden.

Das ökonomische Rechenmodell ist auf die Größen der VGR und den korrespondierenden Input-Output-Tabellen des Statistischen Bundesamts angewiesen. Die VGR gibt u.a. das Arbeitsvolumen und die Zahl der Erwerbstätigen nach 63 Wirtschaftszweigen nach der Wirtschaftszweiggliederung WZ 2008 vor (StBA 2008).

Nationale Prognosemodelle wie INFORGE müssen zwangsläufig mit einer Reihe an Annahmen arbeiten, da nicht alle Variablen endogen bestimmt werden können. Es handelt sich hierbei um Vorgaben für die Entwicklung des Wechselkurses Euro/ Dollar, um die Weltmarktpreisentwicklung von Rohstoffen, den Refinanzierungszins der EZB, um die deutsche Exportnachfrage und um die Bevölkerungsentwicklung in Deutschland.

Wechselkurse und Zinssätze sind - vor allem in der langen Frist (Sarno et al. 2002) - in der Regel schwierig zu projizieren, weshalb es sich in makroökonomischen Prognosemodellen bewährt hat, diese Parameter im Prognosezeitraum auf dem zuletzt bekannten Wert konstant zu halten. Damit nehmen beide Parameter keinen Einfluss auf die Prognose. Dennoch ist es möglich, die Wirkung einer Variation dieser Parameter mittels einer Szenariorechnung zu ermitteln. Weltmarktpreisentwicklung für Rohstoffe wie Erdöl, Erdgas oder Erze basieren auf Vorgaben anderer Institute wie der Internationalen Energieagentur (IEA 2019), dem Internationalen Währungsfonds (IMF 2019) und der Organisation für wirtschaftliche Zusammenarbeit und Entwicklung (OECD und FAO 2019). 
Aufgrund der Exportorientierung Deutschlands ist die Entwicklung und Struktur des Welthandels eine wichtige Einflussgröße für die Entwicklung der deutschen Wirtschaft. Da der Export durch die Importnachfrage der Handelspartner bestimmt wird, ist eine endogene Bestimmung in einem nationalen Modell nicht möglich. Die Exportnachfrage muss daher vorgegeben werden. In INFORGE leitet sie sich aus dem Welthandelsmodell TINFORGE (Mönnig und Wolter 2020) ab. Für die Projektion wird ein durchschnittlicher jährlicher Anstieg des Welthandels zwischen 2020 bis 2040 von 4,7\% angenommen. Die nominalen Exporte Deutschlands wachsen um ca. $2,9 \%$.

Darüber hinaus wird die durchschnittliche Jahresarbeitszeit exogen gesetzt. Langfristig zeigt sich hier ein Trend zurückgehender Jahresarbeitszeiten. Wird das Angebot an Arbeitskräften jedoch zu knapp, muss die durchschnittliche Jahresarbeitszeit geringfügig erhöht werden. In der Basisprojektion wird sie bis zum Jahr 2025 noch leicht sinken, um anschließend bis zum 2035 wieder das heutige Niveau zu erreichen.

\subsection{Die QuBe-Bevölkerungsprojektion}

Der demografische Wandel ist eine entscheidende Einflussgröße auf die ökonomische Entwicklung. Die Bevölkerungsentwicklung wird aus der QuBe-Bevölkerungsprojektion (Fuchs et al. 2017; Kalinowski et al. 2021) entnommen. Spezifika des Bevölkerungsmoduls sind zum einen die Unterscheidung zwischen Deutschen und Nichtdeutschen und zum anderen die Schätzung der einzelnen Komponenten (Geburtenziffern, Überlebenswahrscheinlichkeiten, $\mathrm{Zu}$ - und Abwanderungen sowie Einbürgerungen) mit zeitreihenanalytischen Methoden sowie deren Fortschreibung für die Zukunft (Fuchs et al. 2017; Gorodetski et al. 2016). Die Bevölkerung beeinflusst in INFORGE durch ihre Anzahl und Zusammensetzung nach Altersgruppen die Konsumausgaben und -struktur der privaten Haushalte (Stöver und Wolter 2015), die Ausgaben des Staates und auch die Bauinvestitionen.

Tab. 1 stellt die wesentlichen Größen der Bevölkerungsprojektion dar. Nach den hohen Nettozuwanderungsgewinnen im Jahr 2015 von rund 1,14 Mio. Personen in

Tab. 1 Zentrale Kenngrößen der QuBe-Bevölkerungsprojektion 2015-2040

\begin{tabular}{|c|c|c|c|c|c|c|c|}
\hline \multicolumn{2}{|l|}{ Jahr } & 2015 & 2020 & 2025 & 2030 & 2035 & 2040 \\
\hline \multicolumn{2}{|c|}{ Gesamtbevölkerung in Mio. Personen } & 82,18 & 83,41 & 84,01 & 84,09 & 83,94 & 83,67 \\
\hline \multicolumn{2}{|c|}{ Nichtdeutsche Anteil am Gesamt } & $11 \%$ & $13 \%$ & $14 \%$ & $15 \%$ & $15 \%$ & $16 \%$ \\
\hline \multicolumn{2}{|c|}{ Zuzüge in Mio. Personen } & 2,14 & 1,40 & 1,28 & 1,18 & 1,16 & 1,14 \\
\hline \multicolumn{2}{|c|}{ Fortzüge in Mio. Personen } & 1,00 & 1,00 & 1,00 & 1,00 & 0,99 & 0,99 \\
\hline \multicolumn{2}{|c|}{ Saldo in Mio. Personen } & 1,14 & 0,41 & 0,27 & 0,18 & 0,17 & 0,15 \\
\hline \multirow[t]{2}{*}{$\begin{array}{l}\text { Zusammengefasste } \\
\text { Geburtenziffer }\end{array}$} & $\begin{array}{l}\text { Deutsche } \\
\text { Frauen }\end{array}$ & 1,43 & 1,44 & 1,52 & 1,55 & 1,57 & 1,57 \\
\hline & Ausländerinnen & 1,96 & 2,09 & 2,06 & 2,05 & 2,05 & 2,05 \\
\hline \multirow{2}{*}{$\begin{array}{l}\text { Lebenserwartung in } \\
\text { Jahren bei Geburt }\end{array}$} & Weiblich & 83,06 & 83,80 & 84,80 & 85,61 & 86,19 & 86,68 \\
\hline & Männlich & 78,18 & 78,94 & 79,86 & 80,74 & 81,57 & 82,34 \\
\hline \multirow{2}{*}{$\begin{array}{l}\text { Medianalter in } \\
\text { Jahren }\end{array}$} & Weiblich & 47,19 & 47,48 & 47,06 & 47,24 & 47,63 & 48,06 \\
\hline & Männlich & 44,31 & 43,98 & 43,97 & 44,29 & 44,87 & 44,90 \\
\hline
\end{tabular}

Quelle: QuBe-Projekt, sechste Welle, Bevölkerungsfortschreibung des Statistischen Bundesamtes 
Folge des Syrien-Konfliktes (Zika et al. 2017) ist die Nettozuwanderung in den Folgejahren wieder abgeklungen. Allerdings lag sie in den Jahren 2017 und 2018 mit jeweils rund 400.000 Personen immer noch deutlich über dem langfristigen Durchschnitt von rund 300.000 Personen seit dem Jahr 1991. Die monatliche Wanderungsstatistik des Statistischen Bundesamtes zeigt im ersten Halbjahr 2020 einen starken Rückgang in den Wanderungen, der vermutlich mit den europaweiten Shutdowns in Verbindung steht. Der geschätzte Wanderungssaldo in Höhe von rund 411.000 Personen im Jahr 2020 (siehe Tab. 1) ist deshalb vermutlich zu hoch. Sofern die entsprechenden Wanderungswünsche in den Folgemonaten/-jahren nachgeholt werden, hat diese Überschätzung keine bedeutenden langfristigen Auswirkungen auf die Bevölkerungsprojektion.

In der langfristigen Projektion sinkt die Nettozuwanderung von ihrem hohen Niveau auf rund 150.000 Personen im Jahr $2040 \mathrm{ab}$. Insgesamt wird aufgrund durchgehend positiver Zuwanderungsgewinne bis zum Jahr 2030 ein Bevölkerungswachstum auf rund 84,1 Mio. erwartet. Bis zum Jahr 2040 geht die Bevölkerungszahl wieder auf rund 83,7 Mio. Personen zurück und liegt damit etwas höher als im Jahr 2020.

\subsection{Arbeitsnachfrage nach Wirtschaftszweigen und Berufen}

Die Arbeitsnachfrage $a v b a$ in Stunden und nach Wirtschaftszweigen ist durch die Produktion und den Reallohn bestimmt. Während grundsätzlich eine steigende Realproduktion $y$ positiv auf die Arbeitsnachfrage wirkt, dämpft ein steigender Reallohn $w / p$ den Bedarf. Die Arbeitsnachfrage in Köpfen ergibt sich definitorisch durch die Division des geschätzten Arbeitsvolumens mit der exogen gesetzten Jahresarbeitszeit.

$$
\operatorname{avba}_{i}=\beta_{1}+\beta_{2} \cdot y_{i}+\beta_{3} \cdot \frac{w_{i}}{p_{i}}
$$

Dabei stellt $i=1, \ldots, 63$ den Index für die Wirtschaftszweige dar. Die Arbeitsvolumenquoten $a v b a q$ - definiert als Anteil der Arbeitsstunden einer Berufsgruppe im Wirtschaftszweig - hängen allein von den entsprechenden Lohndifferenzen ab (Maier et al. 2014). Das heißt, die Arbeitsvolumenquote sinkt, wenn der Berufslohn in den 144 Berufsgruppen $o$ im Wirtschaftszweig schneller steigt als der Durchschnittslohn im Wirtschaftszweig insgesamt.

$$
\operatorname{avbaq}_{i, o}=\beta_{1}+\beta_{2} \cdot \frac{w_{i, o}}{w_{i}}
$$

In Kombination ergibt die Schätzung des wirtschaftszweigspezifischen Arbeitsvolumens und die Arbeitsvolumenquoten nach Berufen im Wirtschaftszweig das Arbeitsvolumen $a v$ nach Wirtschaftszweigen und Berufen in Stunden.

$$
a v_{i, o}=\operatorname{avbaq}_{i, o} \cdot \operatorname{avba}_{i}
$$

Die Zahl der Erwerbstätigen $e$ nach Wirtschaftszweig und Beruf ergibt sich per Definition durch Division der Arbeitsvolumen mit der Jahresarbeitszeit. Dabei wird 
die Dynamik der exogen vorgegebenen Jahresarbeitszeit nach Wirtschaftszweigen auf alle Berufsgruppen übertragen.

Die wirtschaftszweigspezifischen Berufslöhne wiederum, die in die Schätzgleichung für die Arbeitsvolumenquoten bestimmend einfließen, ergeben sich aus der Lohnentwicklung in der jeweiligen Berufsgruppe $w_{o}$ und stehen in Abhängigkeit zur wirtschaftszweigspezifischen Produktivität $y / e$. Je produktiver ein Wirtschaftszweig wird, desto höher fällt die Entlohnung aus.

$$
w_{i, o}=\beta_{1}+\beta_{2} \cdot w_{o}+\beta_{3} \cdot \frac{y_{i}}{e_{i}}
$$

Die Berufslohnentwicklung $w_{o}$ wiederum hängt neben dem Durchschnittslohn $W$ von berufsspezifischer Knappheit ab. Das heißt, der Lohn in einer Berufsgruppe nimmt entsprechend $\mathrm{zu}$, je enger das Verhältnis der Erwerbstätigen $e \mathrm{zu}$ den Erwerbspersonen $e p$ ist.

$$
w_{o}=\beta_{1}+\beta_{2} \cdot W+\beta_{3} \cdot \frac{e_{o}}{e p_{o}}
$$

Während die Anzahl der Erwerbstätigen und das Arbeitsvolumen aus der VGR stammt, geht die Information, welche Berufe in welchen Wirtschaftszweigen nachgefragt werden, aus dem Mikrozensus und der Beschäftigtenhistorik der Bundesagentur für Arbeit hervor. Die Strukturinformationen des Mikrozensus dienen dazu, die verschiedenen Datenquellen mittels iterativer Randsummenanpassungsverfahren (Bachem und Korte 1979) so aufeinander abzustimmen, dass die sozialversicherungspflichtig Beschäftigten und ausschließlich geringfügig Beschäftigten nach ausgeübtem Beruf der Beschäftigtenhistorik und die Erwerbstätigen nach Wirtschaftszeig mit den Erwerbstätigen nach VGR übereinstimmen. Die berufsspezifischen Löhne sind Tageslöhne von Vollzeitbeschäftigten aus der Beschäftigtenhistorik (Mönnig et al. 2019). Sie werden nach Schätzung der zensierten Löhne (Büttner und Rässler 2008) direkt aus der Beschäftigtenhistorik entnommen.

\subsection{Arbeitsangebot nach erlerntem Beruf}

Wie bereits im vorherigen Abschnitt abgeleitet, nimmt das Arbeitsangebot auf Berufsgruppenebene Einfluss auf die Lohnentwicklung und somit auch auf die Arbeitsnachfrage im ökonomischen Kernmodell INFORGE. Jedoch wirkt sich auch die ökonomische Entwicklung auf das Arbeitsangebot aus.

Grundlage der Arbeitsangebotsprojektion ist die QuBe-Bevölkerungsprojektion nach Geschlecht, Alter und Nationalität. Über ein Kohorten-Komponentenmodel (Kalinowski et al. 2021) wird das Arbeitsangebot nach erlerntem Beruf ermittelt. Hierfür werden die Bestände von Schülern und Studierenden mit konstanten Übergangsquoten des Jahres 2018 fortgeschrieben. Die Berufswahl stützt sich auf mikrozensusbasierte Auswertungen der Abgänger aus dem beruflichen Bildungssystem nach Berufsgruppen. Erkennbare Entwicklungen in der Berufswahl innerhalb einer 
Qualifikationsstufe ${ }^{3}$ werden mit logarithmierten Trends fortgeschrieben. Die Fortschreibung des Bestandes an Personen außerhalb des Bildungssystems nach Nationalität, Berufsgruppen, Qualifikationen, Geschlecht und Alter ergibt sich durch eine einfache Gleichung: Der Bestand am Jahresende entspricht dem Vorjahresbestand zuzüglich der Nettoübergänge aus dem Bildungssystem, des Wanderungssaldos und abzüglich der Sterbefälle. Für die Bestimmung der zukünftigen Erwerbspersonen wird der Anteil der Erwerbspersonen an der Bevölkerung nach Nationalität, Alter, Qualifikation und Geschlecht mit logarithmierten auslaufenden Trends fortgeschrieben.

Die Erwerbsquoten werden differenziert nach Geschlecht, 14 Altersgruppen und Nationalität (Deutsche/Nichtdeutsche) in Abhängigkeit von autonomen Trends und arbeitsmarktrelevanten Indikatoren (preisbereinigtes Bruttoinlandsprodukt (BIP) pro Kopf, realem Stundenlohn, Erwerbstätigen pro Erwerbsperson sowie Dienstleistungsanteil (Cedefop 2010; Kriechel et al. 2013)) auf Basis des Zeitraums von 1991 bis 2017 geschätzt. Dabei werden alle Schätzansätze einzeln und in unterschiedlichen Kombinationen mit Zeittrends und/oder Dummy-Variablen getestet. Außerdem wurden die Schätzgleichungen auf lag-Strukturen untersucht. Die Schätzgleichungen sind alle logarithmiert. Entsprechen die Vorzeichen den theoretischen, aus der Literatur abgeleiteten Erwartungen einer positiven signifikanten Korrelation (t-Statistik) und es besteht keine Autokorrelation (Durbin-Watson), werden sie in das Model übernommen. In gut einem Drittel der Fälle wird ein Bestimmtheitsmaß von mindestens $80 \%$ erreicht.

Es zeigt sich, dass die konjunkturelle Entwicklung (BIP/Kopf) vor allem bei Frauen (deutsche wie auch nicht deutsche) ein guter Indikator für die Entwicklung der Erwerbsquote ist. Auch der Dienstleistungsanteil weist insbesondere bei den über 40-Jährigen eine hohe Erklärungskraft auf. Die Erwerbstätigenknappheit liefert in Bezug auf Frauen hingegen kaum Erklärung bezüglich ihrer Erwerbsneigung. Der Zeittrend bei den jüngeren weiblichen Altersgruppen zeigt, dass sowohl bei deutschen als auch bei nichtdeutschen Frauen ein (autonomer) Trend zu einer erhöhten Erwerbsneigung besteht. Dies wird auch durch die Erkenntnis bestärkt, dass Reallohnsteigerungen kaum zu einer erhöhten Erwerbsneigung führen. Besonders stark fällt dies bei den ausländischen Frauen auf. Bei den Männern ist die Erwerbslosenquote - vor allem in den mittleren und älteren Jahrgängen - ein gutes Erklärungsmaß - die Wirtschaftsentwicklung hingegen nicht. Insbesondere in den jüngeren Jahrgängen nimmt hingegen der Reallohn positiv Einfluss auf die Erwerbsneigung. Die Erwerbsquoten $E Q$ nach Geschlecht $g$, Alter $a$ und Nationalität $n$ werden mittels eines Schätzansatzes auf die Erwerbsquoten nach Qualifikationen $q$ übertragen (Gl. 7).

$$
E Q_{n, g, a, q}=\beta_{1}+\beta_{2} \cdot E Q_{n, g, a}
$$

\footnotetext{
3 Ohne vollqualifizierenden beruflichen Abschluss/mit beruflichem Abschluss/Aufstiegsfortbildung, Bachelorabschluss oder Fachhochschul-Diplom/Hochschulabschlüsse ohne Bachelorabschluss oder Fachhochschul-Diplom.
} 


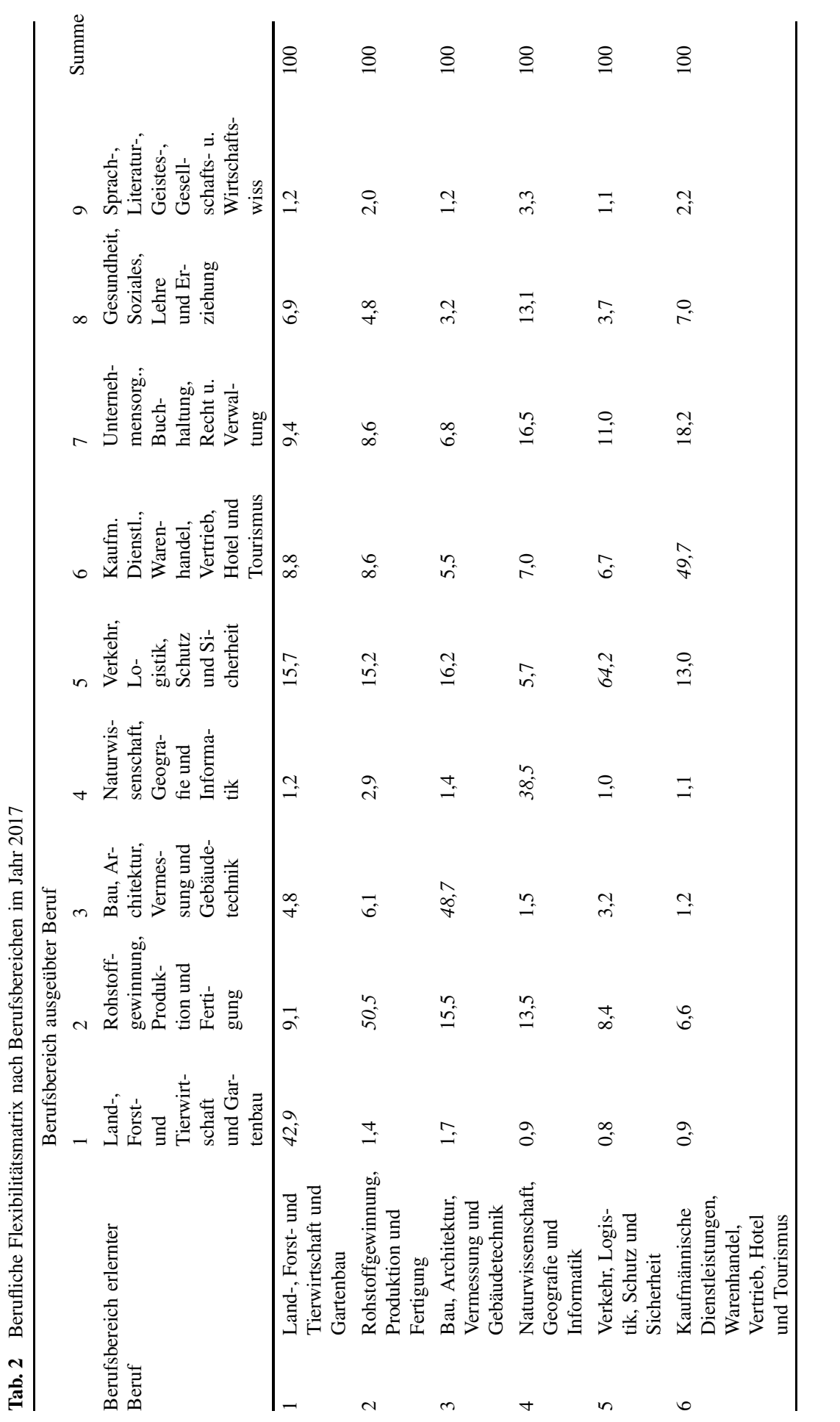




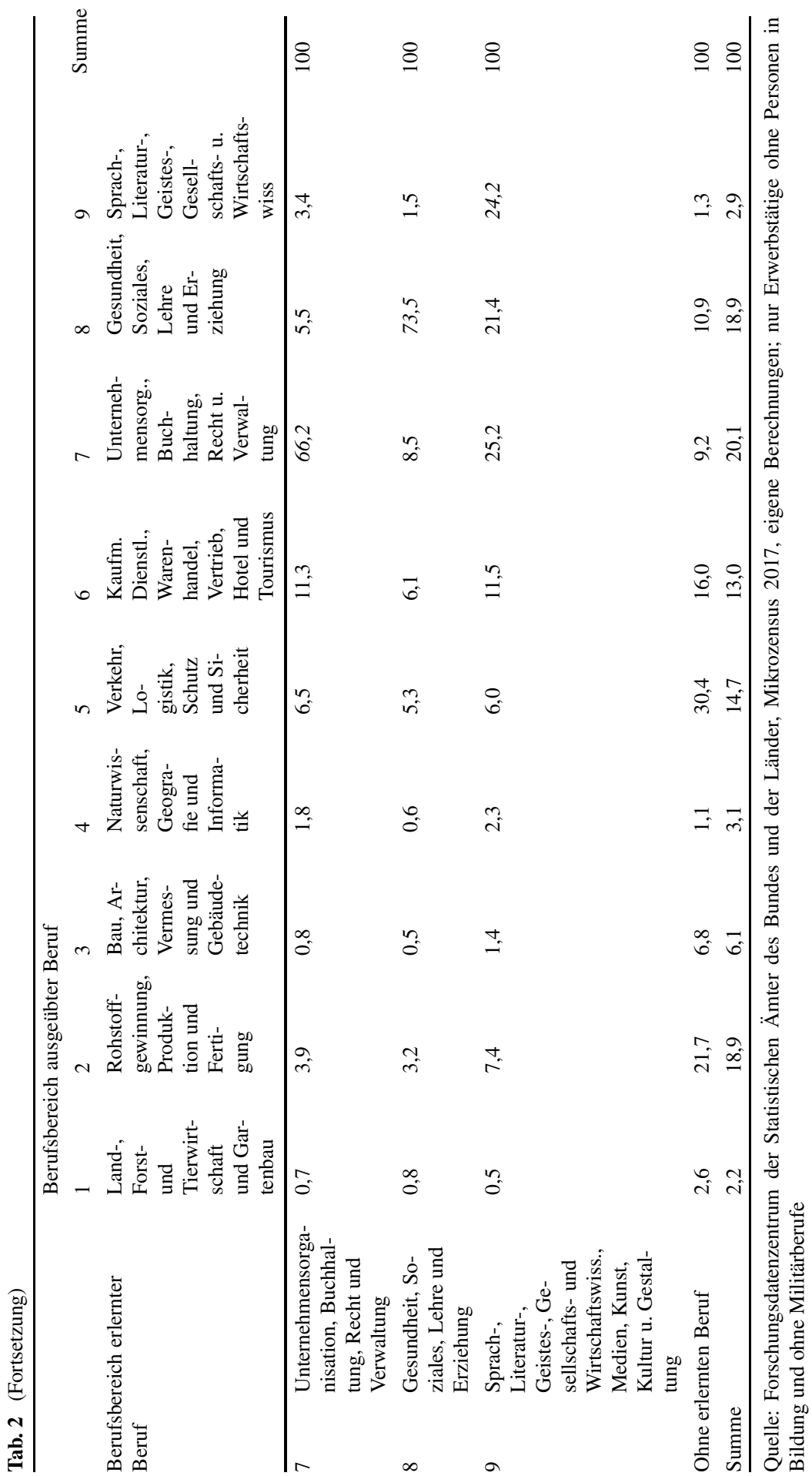


Die Elastizität $\beta_{2}$ gibt wieder, ob die Erwerbsquote bei gleichem Geschlecht, gleicher Nationalität und gleichem Alter entsprechend stärker oder schwächer steigt (vgl. Dunn 1960). Die Wachstumsrate von $E Q_{n, g, a, q}$ wird anschließend auf die Erwerbsquoten nach erlerntem Beruf, differenziert nach 144 Berufsgruppen, übertragen.

\subsection{Berufliche Mobilität - Arbeitsangebot nach ausgeübten Berufen}

Für eine Bilanzierung von Arbeitskräftebedarf und -angebot nach Berufen ist jedoch nicht das Angebot nach erlerntem Beruf ausschlaggebend, sondern jenes Arbeitsangebot, welches unter Berücksichtigung beruflicher Mobilitätsprozesse zustande kommt. Diese berufliche Mobilität wird in Mobilitätsmatrizen widergespiegelt. Sie geben die Wahrscheinlichkeit einer Erwerbstätigkeit in einem Erwerbsberuf, in Abhängigkeit vom höchsten erlernten beruflichen Abschluss wieder (Maier und Helmrich 2012). Sie liegen getrennt nach Nationalität, Geschlecht, Alter (15 bis 34 Jahre, 35 bis 49 Jahre und über 50 Jahre) und vier Qualifikationsstufen für jeweils 144 Berufsgruppen vor. Diese Matrizen sind Ergebnis vergangener Mobilitätsprozesse und spiegeln somit zum einen die Tätigkeitsmöglichkeiten in Abhängigkeit vom erlernten beruflichen Abschluss wider. Zum anderen ist das Ausmaß an beruflicher Flexibilität aber auch das Ergebnis vergangener Marktchancen und -risiken. Tab. 2 zeigt die berufliche Mobilitätsmatrix, aggregiert aus allen Bevölkerungsgruppen, des Jahres 2017 nach Berufsbereichen (Einsteller der KldB 2010). Auf der Diagonalen der Tabelle ist der Anteil der Personen eingetragen, die in ihrem erlernten Berufssegment erwerbstätig sind. Bei den „Sprach-, Literatur-, Geistes-, Gesellschaftsund Wirtschaftswissenschaften, Medien, Kunst, Kultur und Gestaltung" ist dieser Steher-Anteil mit rund 24,2\% am geringsten. Rund 25,2\% wandern in den Berufsbereich „Unternehmensorganisation, Buchhaltung, Recht und Verwaltung“ ab, weitere $21,2 \%$ in den Bereich „Gesundheit, Soziales, Lehre und Erziehung“. Im letztgenannten Berufsbereich ist der Steheranteil mit rund 73,5\% am höchsten. Personen ohne erlernten Beruf sind mit 30,4\% am häufigsten in „Verkehr, Logistik, Schutz und Sicherheit" erwerbstätig.

Für die Projektion wird unterstellt, dass die beobachteten Mobilitäten auch für die Zukunft Bestand haben. Mit einer langfristigen Veränderung des Arbeitsangebotes nach den genannten Bevölkerungsgruppen verändert sich somit auch das entsprechende Arbeitsangebot für die mit den jeweiligen erlernten Berufen ausübbaren Tätigkeiten. Um die Veränderung von Marktchancen gleichermaßen zu berücksichtigen, wird auch die Beziehung zwischen der berufsspezifischen Lohnentwicklung und der Veränderung des Steher-Anteils modelliert (Maier et al. 2017). So wird für den Zeitraum von 2005 bis 2017 geprüft, ob der Steher-Anteil stayer ${ }_{o}$ in einer Berufsgruppe auf Veränderungen der berufsspezifischen Entlohnungsmöglichkeiten reagiert hat (Gl. 8).

$$
\text { stayer }_{o}=\delta_{1}+\delta_{2} \frac{w_{o}}{w_{o}^{\text {ref }}}, \text { mit } o=144 \text { Berufsgruppen }
$$

Dabei stellt $w_{o}$ den Lohn in der Berufsgruppe dar und $w_{o}^{\text {ref }}$ gibt den Referenzlohn wieder. Dies ist der Durchschnittlohn, den eine Person mit ihrer beruflichen 
Qualifizierung erhalten kann. Er berechnet sich aus den Durchschnittslöhnen aller Berufsgruppen, gewichtet mit den entsprechenden Mobilitätsanteilen. In Summe kann in 74 von 144 Berufsgruppen zwischen 2005 und 2017 nachgewiesen werden, dass sich mit einer relativen Erhöhung/Verringerung des Berufslohns auch der Anteil der Steher erhöht/verringert hat. Für zehn weitere Berufsordnungen wird die Elastizität der übergeordneten Berufshauptgruppen auf die darunterliegenden Berufsgruppen übertragen. Für diese Berufe wird auch in Zukunft unterstellt, dass eine Anpassungsreaktion des Arbeitsangebotes stattfindet, wenn sich die berufsspezifischen Löhne (siehe Gl. 5) verändern. Damit ergibt sich bei einer veränderten Nachfrage nach Berufen auch ein verändertes Angebot an Erwerbspersonen, die für die jeweiligen Berufe zur Verfügung stehen, was wiederum Rückwirkungen auf den berufsspezifischen Lohn (siehe Gl. 6) hat.

\section{Auswirkungen der COVID-19-Pandemie}

Um die Anpassungsprozesse von QINFORGE zu illustrieren, vergleichen wir ein kontrafaktisches Szenario, welches ausgehend vom Jahr 2019 die empirisch erfassten Trends und Verhaltensweisen in die Zukunft projiziert (Maier et al. 2020), mit einer Basisprojektion, welche den durch die Corona-Pandemie bedingten Wirtschaftseinbruch (StBA 2020) abbildet. Tab. 3 zeigt in einem Überblick die Unterschiede zwischen einer Welt ohne Corona-Krise und der QuBe-Basisprojektion, welche die vermuteten Auswirkungen der Corona-Pandemie in der kurzen und mittleren Frist berücksichtigt. Die Basisprojektion stützt sich dabei auf Erkenntnisse von Umfrageund Registerdaten mit Stand September 2020. Nicht in Tab. 3 aufgeführt, aber Teil der Basisprojektion sind die stützenden Maßnahmen in Höhe von $130 \mathrm{Mrd}$ €, die durch das Konjunkturpaket der Bundesregierung am 3. Juni 2020 aktiviert wurden.

Tab. 3 Eingriffe in QINFORGE zur Modellierung der Auswirkungen der COVID-19-Pandemie

\begin{tabular}{|c|c|}
\hline Exporte & Rückgang über alle Wirtschaftszweige wegen einbrechendem Welthandel \\
\hline Importe & $\begin{array}{l}\text { Weniger Importe für Pharma- und Textilindustrie, da mehr im Inland hergestellt } \\
\text { wird }\end{array}$ \\
\hline Investitionen & Investitionsattentismus aufgrund von Unsicherheit \\
\hline $\begin{array}{l}\text { Gesundheits- } \\
\text { ausgaben }\end{array}$ & Steigende Ausgaben wegen Pandemie \\
\hline $\begin{array}{l}\text { Konsum der pri- } \\
\text { vaten Haushalte }\end{array}$ & $\begin{array}{l}\text { Weniger Nachfrage aufgrund des Shutdowns vor allem bei Verwendungszwecken } \\
\text { wie „Möbel, Innenausstattung, Teppiche u. ä.“, „Pauschalreisen“, „Verpflegungs- } \\
\text { dienstleistungen“, „Beherbergungsdienstleistungen“ und „Freizeit und Kulturdienst- } \\
\text { leistungen“ } \\
\text { Stärkere Nachfrage nach dem Verwendungszweck „Telefon- und Telefaxdienstleis- } \\
\text { tungen, Internet“” }\end{array}$ \\
\hline Arbeitsvolumen & Stundenreduktion für das Gastgewerbe \\
\hline $\begin{array}{l}\text { Bestand an } \\
\text { Personenkraft- } \\
\text { wagen }\end{array}$ & Rückgang \\
\hline Ölpreis & Rückgang \\
\hline Jahresarbeitszeit & Verringerung der Jahresarbeitszeit aufgrund geringerer Arbeitskräfteknappheit \\
\hline
\end{tabular}




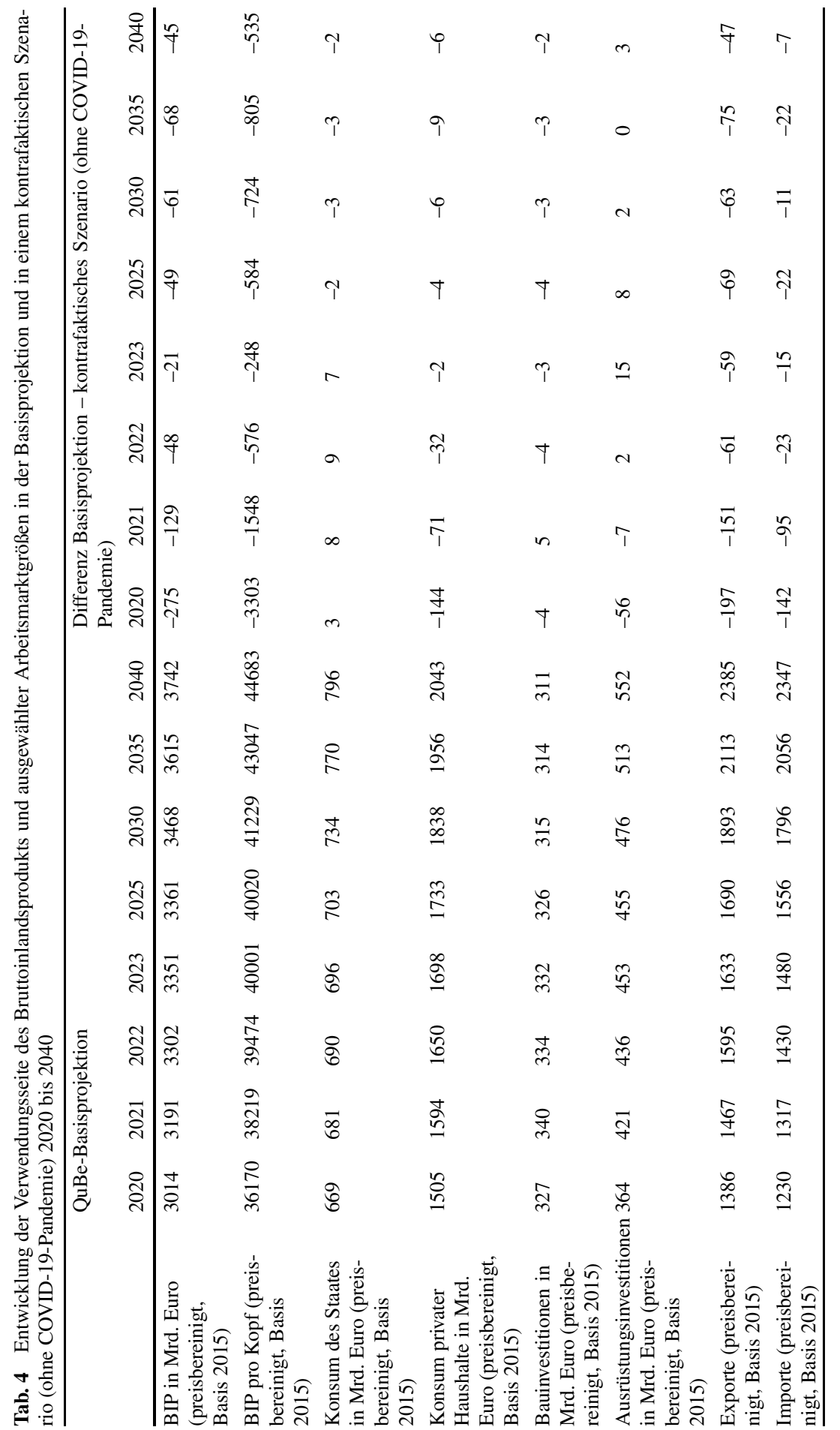




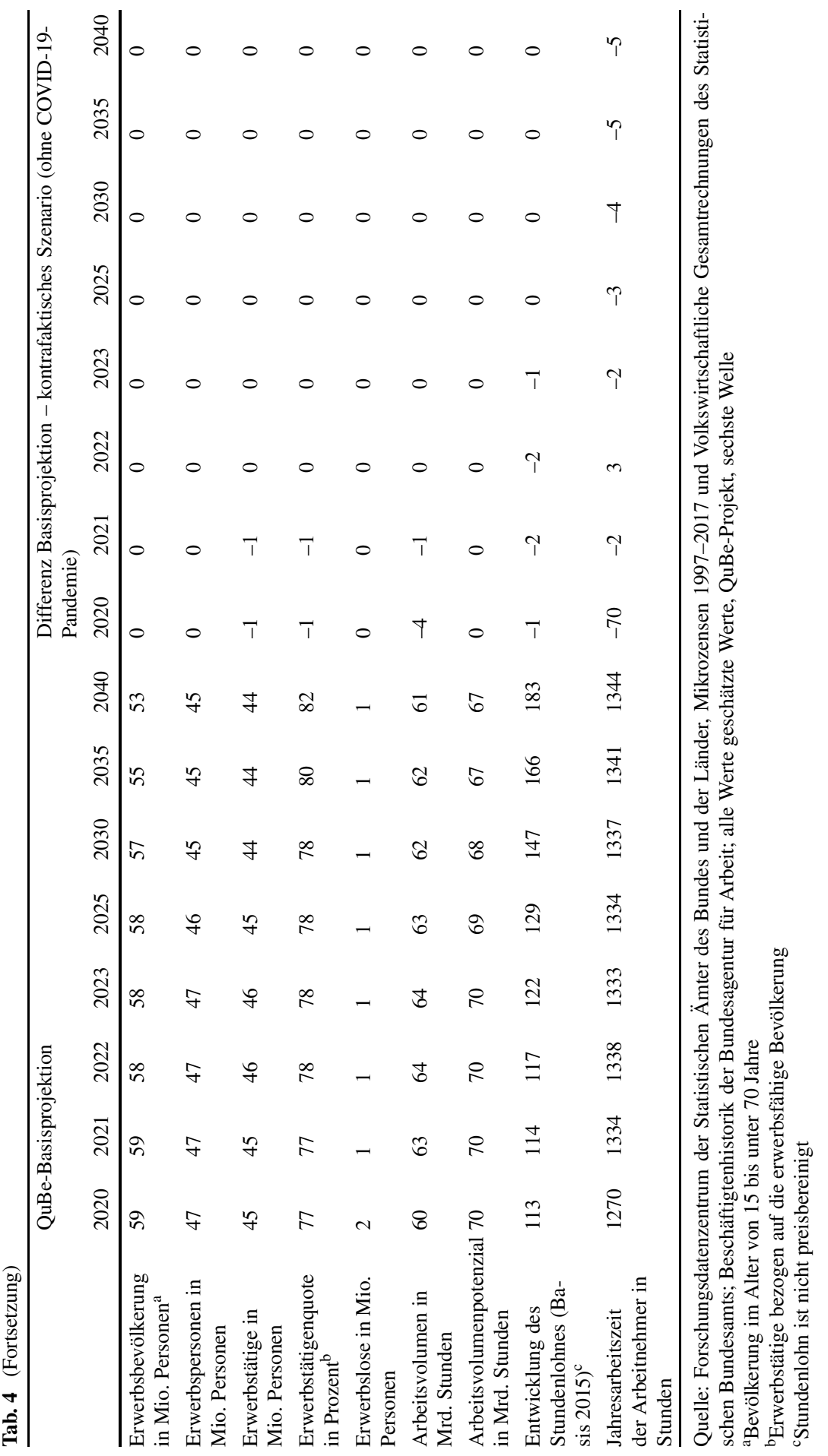


Für die Vielzahl der hierfür notwendigen Annahmen sei auf Wolter et al. (2020) verwiesen. Die Unterschiede zwischen dem kontrafaktischen Szenario und der QubeBasisprojektion werden im folgenden Abschnitt aufgezeigt.

\section{Ergebnisse}

\section{1 Ökonomische Entwicklung}

Tab. 4 enthält die Kenngrößen der ökonomischen Entwicklung und des Arbeitsmarktes der QuBe-Basisprojektion sowie die Differenz der Größen zum kontrafaktischen Szenario. Ohne die Einschränkungen der Corona-Pandemie wäre für das Jahr 2020 ein Wachstum des preisbereinigten Bruttoinlandsproduktes (BIP) in Höhe von 1,5\% möglich gewesen. Die durchschnittliche Wachstumsrate von 2020 bis 2040 hätte rund $0,7 \%$ betragen. In der Basisprojektion wird im Jahr 2020 aufgrund des „Shutdowns“ und dem damit verbundenen Wirtschaftseinbruch ein Rückgang des preisbereinigten BIP im Vergleich zum Jahr 2019 in Höhe von 7,0\% errechnet. ${ }^{4}$ Dies ist auf die geringere Investitionsneigung, den schrumpfenden Welthandel mit geringeren Exporten aus Deutschland, Produktionsstopps und zerrissene Lieferketten zurückzuführen. Die Schließungen zur Reduktion von Infektionsketten haben zudem stark negative Wirkungen auf den Konsum der privaten Haushalte vor allem in den Bereichen Freizeit, Tourismus, Kultur und Sport. Ausgebliebene Reisetätigkeiten und die geringeren Konsummöglichkeiten aufgrund geringeren Einkommens (Kurzarbeit) tragen überdies zum Rückgang bei. Die höheren Konsumausgaben des Staates in der Basisprojektion im Vergleich zum kontrafaktischen Szenario zeigen bereits die Folgen des Konjunkturpaketes (Wolter et al. 2020).

Die Erholungsphase des Exports erstreckt sich entsprechend den Modellrechnungen aufgrund der weltweiten Unsicherheiten und unterschiedlichen Betroffenheit durch das Corona-Virus hingegen auf rund zwei Jahre (,langes V“). Unabhängig von der Corona-Pandemie sind die Risiken im Außenhandel - vor allem aufgrund der schwer einzuschätzenden Handelspolitik der USA - deutlich gestiegen. Ab dem Jahr 2025 ähneln die Wachstumsraten der Basisprojektion den erwarteten Wachstumsraten ohne Corona-Pandemie (kontrafaktisches Szenario). Der Wohlstand, der ohne die derzeitige Krise womöglich erreicht worden wäre, wird jedoch voraussichtlich erst mit einer ca. dreijährigen Verzögerung erreicht.

\footnotetext{
${ }^{4}$ Das tatsächliche Wirtschaftswachstum fiel mit $-5 \%$ um rund zwei Prozentpunkte geringer aus. Die Wachstumsprognose des preisbereinigten BIP von 2020 auf 2021 liegt mit rund 5,9\% hingegen um rund zwei Prozentpunkte oberhalb der Prognosen anderer Institutionen (Bundesministerium für Wirtschaft und Energie 2021: 3,0\%; OECD 2020: 2,8\%; Deutsche Bundesbank 2020: 3,0\%). Ab dem Jahr 2022 ähneln die Wachstumserwartungen der Basisprojektion den anderen Projektionen. Die substanziellen Aussagen des Szenariovergleichs verändern sich durch diese abweichenden Entwicklungen nicht.
} 


\subsection{Entwicklung des Arbeitsmarktes}

Am Arbeitsmarkt zeigt sich in der Basisprojektion vor allem ein starker Rückgang des Arbeitsvolumens in Höhe von 5,5 \% im Jahr 2020.5 Im Jahr 2021 ist das Arbeitsvolumen allerdings fast wieder auf der Höhe des Jahres 2019. Trotz der möglichen Kurzarbeit wird aufgrund der Corona-Pandemie auch die Erwerbslosigkeit ansteigen. Selbst wenn betriebsbedingte Kündigungen oftmals vermieden werden können, so werden freiwerdende Stellen aufgrund der Unsicherheiten zunächst nicht wiederbesetzt (Hutter und Weber 2020). In den Jahren 2020 und 2021 werden jeweils rund 600.000 Erwerbstätige weniger benötigt als dies im kontrafaktischen Szenario der Fall gewesen wäre. Da sich aufgrund der schlechteren Arbeitsmarktlage auch einige Erwerbspersonen vom Arbeitsmarkt zurückziehen werden (siehe Abschn. 2.5), steigt die Zahl der Erwerbslosen in beiden Jahren mit rund 400.000 Personen im Vergleich zum kontrafaktischen Szenario geringer an. ${ }^{6}$

Die zunehmende Unterbeschäftigung ist in der Entwicklung des Stundenlohns und der Jahresarbeitszeit der Beschäftigten berücksichtigt: So hemmt die CoronaPandemie vor allem in der kurzen und mittleren Frist das Lohnwachstum, welches in einem durch Fachkräfteengpässe gekennzeichneten Arbeitsmarkt im kontrafaktischen Szenario möglich gewesen wäre. In einer ,Welt ohne Corona-Virus“ hätten die Arbeitnehmer auch mehr Arbeitsstunden anbieten müssen, um die Arbeitsnachfrage befriedigen zu können. In der Basisprojektion ist eine Steigerung der Jahresarbeitszeiten zwar ebenfalls notwendig, jedoch in einer etwas geringeren Dynamik. Das geringere Lohnwachstum sowie die geringeren durchschnittlichen Arbeitsstunden führen dazu, dass in der Basisprojektion zum Projektionsende im Jahr 2040 genauso viele Personen in Erwerbstätigkeit sein werden, wie es im kontrafaktischen Szenario der Fall gewesen wäre.

\subsection{Berufsspezifische Auswirkungen}

Tab. 5 stellt für die Jahre von 2020 bis 2040 die Berufsgruppen (Dreisteller der KldB 2010) dar, welche im Vergleich der Basisprojektion zum kontrafaktischen Szenario die höchsten prozentualen Abweichungen in der Erwerbstätigkeit aufweisen. Da die Erwerbstätigkeit in den meisten Berufsgruppen aufgrund der COVID-19-Pandemie zurückgeht, sind die 20 Berufsgruppen mit den höchsten Erwerbstätigenverlusten dargestellt - hingegen nur 10 Berufsgruppen mit dem höchsten Zuwachs im Bedarf an Erwerbstätigen. Die Sortierung erfolgt anhand des Jahres 2020. Dabei werden nur Berufsgruppen betrachtet, die auch mindestens 20.000 Erwerbstätige stellen.

Für den Bedarf an Erwerbstätigen in den Berufsgruppen ist die Arbeitsnachfrage in Stunden in den jeweiligen Branchen ausschlaggebend. Die Berufsgruppen sind deshalb entsprechend ihrer Verteilung auf die Branchen betroffen, auch wenn

\footnotetext{
5 Tatsächlich gingen die geleisteten Arbeitsstunden um 4,8\% zurück.

${ }^{6}$ Für das Jahr 2020 weist die Volkswirtschaftliche Gesamtrechnung eine um 474.000 Personen höhere Anzahl an Erwerbslosen aus als 2019. Die Anzahl der erwerbstätigen Inländer liegt im Jahr 2020 um 432.000 Personen unter dem Wert des Jahres 2019. Ohne COVID-19-Krise wäre jedoch auch ein weiteres Erwerbstätigenwachstum im Vergleich zu 2019 möglich gewesen.
} 
Tab. 5 Berufsgruppen mit den 20 stärksten Rückgängen und zehn stärksten Zuwächsen in der Erwerbstätigkeit aufgrund der COVID-19-Pandemie (Basisprojektion - kontrafaktisches Szenario) 2020 in Prozent und Anpassung bis 2040

\begin{tabular}{|c|c|c|c|c|c|c|c|c|c|}
\hline \multicolumn{2}{|c|}{ Berufsgruppe } & \multicolumn{8}{|l|}{ Jahr } \\
\hline & & 2020 & 2021 & 2022 & 2023 & 2025 & 2030 & 2035 & 2040 \\
\hline 633 & Gastronomie & -12 & -11 & -5 & -1 & -2 & -2 & -1 & 0 \\
\hline 632 & Hotellerie & -11 & -11 & -5 & -1 & -2 & -2 & -1 & 0 \\
\hline 631 & Tourismus und Sport & -10 & -10 & -4 & -1 & 0 & 0 & 0 & 0 \\
\hline 293 & Speisenzubereitung & -10 & -9 & -4 & -1 & -1 & -2 & 0 & 0 \\
\hline 941 & $\begin{array}{l}\text { Musik-, Gesangs- und } \\
\text { Dirigententätigkeiten }\end{array}$ & -8 & -7 & -3 & 0 & 0 & -1 & -1 & -1 \\
\hline 942 & $\begin{array}{l}\text { Schauspiel, Tanz und } \\
\text { Bewegungskunst }\end{array}$ & -7 & -6 & -3 & 0 & 0 & 0 & 0 & 0 \\
\hline 933 & $\begin{array}{l}\text { Kunsthandwerk und } \\
\text { bildende Kunst }\end{array}$ & -7 & -6 & -3 & 0 & 0 & -1 & -1 & 0 \\
\hline 514 & $\begin{array}{l}\text { Servicekräfte im Perso- } \\
\text { nenverkehr }\end{array}$ & -6 & -4 & -2 & -1 & -1 & -1 & -1 & -1 \\
\hline 943 & $\begin{array}{l}\text { Moderation und Unter- } \\
\text { haltung }\end{array}$ & -6 & -5 & -3 & 0 & 0 & 0 & 0 & 0 \\
\hline 845 & $\begin{array}{l}\text { Fahr- und Sportunter- } \\
\text { richt an außerschul. } \\
\text { Bildungseinrichtungen }\end{array}$ & -4 & -1 & 0 & 1 & 1 & 0 & 0 & 0 \\
\hline 945 & $\begin{array}{l}\text { Veranstaltungs-, Kame- } \\
\text { ra- und Tontechnik }\end{array}$ & -4 & -4 & -3 & -2 & -1 & -1 & -1 & -1 \\
\hline 844 & $\begin{array}{l}\text { Lehrtätigkeit an außer- } \\
\text { schulischen Bildungs- } \\
\text { einrichtungen }\end{array}$ & -4 & -1 & 0 & 1 & 1 & 0 & -1 & -1 \\
\hline 944 & $\begin{array}{l}\text { Theater-, Film- und } \\
\text { Fernsehproduktion }\end{array}$ & -3 & -5 & -3 & -2 & -2 & -1 & 0 & 0 \\
\hline 634 & $\begin{array}{l}\text { Veranstaltungsservice } \\
\text { und -management }\end{array}$ & -3 & -4 & -2 & -1 & -1 & -1 & 0 & 0 \\
\hline 623 & $\begin{array}{l}\text { Verkauf von Lebens- } \\
\text { mitteln }\end{array}$ & -3 & -3 & -1 & 0 & 0 & 0 & 0 & 0 \\
\hline 733 & $\begin{array}{l}\text { Medien-, Dokumenta- } \\
\text { tions- und Informati- } \\
\text { onsd. }\end{array}$ & -3 & -2 & -1 & 1 & 0 & 0 & 0 & 0 \\
\hline 511 & $\begin{array}{l}\text { Techn. Betrieb des } \\
\text { Eisenbahn-, Luft- und } \\
\text { Schiffsverkehrs }\end{array}$ & -3 & -2 & -1 & 0 & -1 & -1 & -1 & -1 \\
\hline 924 & $\begin{array}{l}\text { Redaktion und Journa- } \\
\text { lismus }\end{array}$ & -3 & -3 & -2 & -1 & -1 & -1 & 0 & -1 \\
\hline 292 & $\begin{array}{l}\text { Lebensmittel- und Ge- } \\
\text { nussmittelherstellung }\end{array}$ & -3 & -2 & 0 & 0 & 0 & 0 & 0 & 0 \\
\hline 721 & $\begin{array}{l}\text { Versicherungs- und } \\
\text { Finanzdienstleistungen }\end{array}$ & -2 & -2 & -1 & -1 & -1 & -1 & -1 & -1 \\
\hline$\cdots$ & & & & & & & & & \\
\hline 112 & Tierwirtschaft & 1 & 1 & 1 & 1 & 0 & 0 & 0 & 1 \\
\hline 832 & $\begin{array}{l}\text { Hauswirtschaft und } \\
\text { Verbraucherberatung }\end{array}$ & 1 & 1 & 1 & 1 & 0 & 0 & 1 & 1 \\
\hline
\end{tabular}


Tab. 5 (Fortsetzung)

\begin{tabular}{|c|c|c|c|c|c|c|c|c|c|}
\hline & \multicolumn{8}{|l|}{ Jahr } \\
\hline \multicolumn{2}{|c|}{ Berufsgruppe } & 2020 & 2021 & 2022 & 2023 & 2025 & 2030 & 2035 & 2040 \\
\hline 843 & $\begin{array}{l}\text { Lehr- und Forschungs- } \\
\text { tätigkeit an Hochschu- } \\
\text { len }\end{array}$ & 1 & 2 & 3 & 4 & 5 & 4 & 4 & 4 \\
\hline 816 & $\begin{array}{l}\text { Psychologie und nicht } \\
\text { ärztliche Psychothera- } \\
\text { pie }\end{array}$ & 2 & 2 & 2 & 2 & 0 & 0 & 0 & 0 \\
\hline 812 & $\begin{array}{l}\text { Medizinisches Labora- } \\
\text { torium }\end{array}$ & 2 & 2 & 2 & 2 & 0 & 0 & 0 & 0 \\
\hline 817 & $\begin{array}{l}\text { Nicht ärztliche Thera- } \\
\text { pie und Heilkunde }\end{array}$ & 2 & 2 & 2 & 2 & 0 & 0 & 0 & 0 \\
\hline 811 & Arzt- und Praxishilfe & 2 & 2 & 2 & 2 & 1 & 0 & 0 & 0 \\
\hline 814 & $\begin{array}{l}\text { Human- und Zahnme- } \\
\text { dizin }\end{array}$ & 2 & 2 & 3 & 2 & 1 & 0 & 0 & 0 \\
\hline 813 & $\begin{array}{l}\text { Gesundheits- und Kran- } \\
\text { kenpflege, Rettungsd. } \\
\text { u. Geburtshilfe }\end{array}$ & 3 & 1 & 2 & 2 & 0 & 0 & 0 & 0 \\
\hline 821 & Altenpflege & 5 & 0 & 0 & 0 & 0 & 0 & 0 & 0 \\
\hline
\end{tabular}

Quelle: Forschungsdatenzentrum der Statistischen Ämter des Bundes und der Länder, Mikrozensen 1997-2017 und Volkswirtschaftliche Gesamtrechnungen des Statistischen Bundesamts; Beschäftigtenhistorik der Bundesagentur für Arbeit; Berechnung und Darstellung QuBe-Projekt, sechste Welle; nur Berufsgruppen mit mindestens 20.000 Erwerbstätigen

ihre explizite Tätigkeit vielleicht mehr oder weniger gefragt ist. Am stärksten ist der Rückgang im jeweils zweistelligen Ausmaß in den Berufsgruppen „Gastronomie“, „Hotellerie“, „Tourismus und Sport“ und „Speisenzubereitung“. Zuwächse erwartet das Modell in den Berufen des Gesundheitsbereichs sowie in „Lehr- und Forschungstätigkeiten an Hochschulen“. Dies ist eine Folge des Konjunkturpaketes, welches eine mittelfristige staatliche Förderung für das Bildungswesen sowie für Forschung und Entwicklung vorsieht. In allen anderen in Tab. 5 genannten Berufsgruppen nähert sich die Erwerbstätigkeit bis zum Jahr 2025 wieder dem Niveau der Basisprojektion an.

Tab. 6 zeigt, dass in den Berufsgruppen mit einer zurückgehenden Erwerbstätigkeit auch ein geringeres Lohnwachstum zu erwarten ist, als es ohne die COVID19-Pandemie der Fall gewesen wäre. Allerdings zeigt sich ebenfalls bis zum Jahr 2025 ein Aufholeffekt. Für die Berufe des Gesundheitsbereichs erwartet das Modell ein höheres Lohnniveau im Jahr 2020 als ohne Einfluss der Krise. Allerdings führen die Budgetrestriktionen im Gesundheitsbereich auch dazu, dass es sich bei den Lohnsteigerungen lediglich um einen Vorzieheffekt handelt. Ab dem Jahr 2023 wird in QINFORGE in der Basisprojektion für diese Berufsgruppen dasselbe nominale Lohnniveau prognostiziert wie im kontrafaktischen Szenario. Lediglich in „Lehr- und Forschungstätigkeiten an Hochschulen“ zeigt sich in der Basisprojektion langfristig ein höheres Lohnniveau.

Für die Veränderung des Arbeitsangebotes nach Berufen sind zwei Größen entscheidend (vgl. Abschn. 2.5 und 2.6): zum einen die Erwerbsquoten, die sich aufgrund der unterschiedlichen Konjunktur in beiden Szenarien voneinander unterschei- 
Tab. 6 Lohnveränderungen der Berufsgruppen mit den 20 stärksten Rückgängen und zehn stärksten Zuwächsen in der Erwerbstätigkeit aufgrund der COVID-19-Pandemie (Basisprojektion - kontrafaktisches Szenario) 2020 in Prozent und Anpassung bis 2040

\begin{tabular}{|c|c|c|c|c|c|c|c|c|c|}
\hline & \multirow{2}{*}{\multicolumn{8}{|c|}{ Jahr }} \\
\hline Berufsgruppe & & & & & & & & & \\
\hline 633 & Gastronomie & $\frac{2020}{-7}$ & $\frac{2021}{-7}$ & $\frac{2022}{-2}$ & $\frac{2023}{1}$ & $\frac{2025}{1}$ & $\frac{2030}{1}$ & $\frac{2035}{0}$ & $\frac{2040}{0}$ \\
\hline 632 & Hotellerie & -4 & -5 & -2 & 0 & 1 & 1 & 1 & 0 \\
\hline 631 & Tourismus und Sport & -5 & -7 & -3 & -1 & 1 & 0 & 0 & 0 \\
\hline 293 & Speisenzubereitung & -6 & -6 & -2 & 1 & 1 & 1 & 0 & 0 \\
\hline 941 & $\begin{array}{l}\text { Musik-, Gesangs- und } \\
\text { Dirigententätigkeiten }\end{array}$ & -5 & -6 & -3 & 0 & 1 & 0 & 0 & 0 \\
\hline 942 & $\begin{array}{l}\text { Schauspiel, Tanz und } \\
\text { Bewegungskunst }\end{array}$ & -3 & -4 & -2 & 0 & 1 & 0 & 0 & 0 \\
\hline 933 & $\begin{array}{l}\text { Kunsthandwerk und } \\
\text { bildende Kunst }\end{array}$ & -5 & -7 & -4 & 0 & 1 & 0 & 0 & 0 \\
\hline 514 & $\begin{array}{l}\text { Servicekräfte im Perso- } \\
\text { nenverkehr }\end{array}$ & -2 & -2 & -1 & 0 & 0 & 0 & 0 & 0 \\
\hline 943 & $\begin{array}{l}\text { Moderation und Unter- } \\
\text { haltung }\end{array}$ & -3 & -4 & -2 & 0 & 1 & 0 & 0 & 0 \\
\hline 845 & $\begin{array}{l}\text { Fahr- und Sportunter- } \\
\text { richt an außerschul. } \\
\text { Bildungseinricht }\end{array}$ & -2 & -3 & -2 & 0 & 1 & 0 & 0 & 0 \\
\hline 945 & $\begin{array}{l}\text { Veranstaltungs-, Kame- } \\
\text { ra- und Tontechnik }\end{array}$ & -1 & -3 & -2 & -1 & 0 & 0 & 0 & 0 \\
\hline 844 & $\begin{array}{l}\text { Lehrtätigkeit an außer- } \\
\text { schulischen Bildungs- } \\
\text { einrichtungen }\end{array}$ & -2 & -2 & -2 & 0 & 1 & 0 & 0 & 0 \\
\hline 944 & $\begin{array}{l}\text { Theater-, Film- und } \\
\text { Fernsehproduktion }\end{array}$ & -2 & -5 & -4 & -2 & 0 & 0 & 0 & 0 \\
\hline 634 & $\begin{array}{l}\text { Veranstaltungsservice } \\
\text { und -management }\end{array}$ & -2 & -4 & -3 & -1 & 0 & 0 & 0 & 0 \\
\hline 623 & $\begin{array}{l}\text { Verkauf von Lebens- } \\
\text { mitteln }\end{array}$ & -1 & -4 & -3 & -1 & 1 & 0 & 0 & 0 \\
\hline 733 & $\begin{array}{l}\text { Medien-, Dokumentati- } \\
\text { ons- und Informationsd. }\end{array}$ & -1 & -2 & -2 & 0 & 0 & 0 & 0 & 0 \\
\hline 511 & $\begin{array}{l}\text { Techn. Betrieb des } \\
\text { Eisenbahn-, Luft- und } \\
\text { Schiffsverkehrs }\end{array}$ & -1 & -2 & -1 & 0 & 0 & 0 & 0 & 0 \\
\hline 924 & $\begin{array}{l}\text { Redaktion und Journa- } \\
\text { lismus }\end{array}$ & 0 & -1 & 0 & 0 & 0 & 0 & 0 & 0 \\
\hline 292 & $\begin{array}{l}\text { Lebensmittel- und Ge- } \\
\text { nussmittelherstellung }\end{array}$ & -1 & -3 & -2 & -1 & 0 & 0 & 0 & 0 \\
\hline 721 & $\begin{array}{l}\text { Versicherungs- und } \\
\text { Finanzdienstleistungen }\end{array}$ & -1 & -3 & -3 & -1 & 0 & 0 & 0 & 0 \\
\hline$\ldots$ & & & & & & & & & \\
\hline 112 & Tierwirtschaft & 1 & -2 & -3 & -1 & 0 & 0 & 0 & 0 \\
\hline 832 & $\begin{array}{l}\text { Hauswirtschaft und } \\
\text { Verbraucherberatung }\end{array}$ & 1 & -1 & -2 & -1 & 0 & 0 & 0 & 0 \\
\hline
\end{tabular}


Tab. 6 (Fortsetzung)

\begin{tabular}{|c|c|c|c|c|c|c|c|c|c|}
\hline & \multicolumn{8}{|l|}{ Jahr } \\
\hline \multicolumn{2}{|c|}{ Berufsgruppe } & \multirow{2}{*}{$\frac{2020}{1}$} & \multirow{2}{*}{$\frac{2021}{-1}$} & \multirow{2}{*}{$\frac{2022}{0}$} & \multirow{2}{*}{$\frac{2023}{2}$} & \multirow{2}{*}{$\begin{array}{l}2025 \\
3\end{array}$} & \multirow{2}{*}{$\frac{2030}{3}$} & \multirow{2}{*}{$\frac{2035}{2}$} & \multirow{2}{*}{$\frac{2040}{2}$} \\
\hline 843 & $\begin{array}{l}\text { Lehr- und Forschungs- } \\
\text { tätigkeit an Hochschu- } \\
\text { len }\end{array}$ & & & & & & & & \\
\hline 816 & $\begin{array}{l}\text { Psychologie und nicht } \\
\text { ärztliche Psychothera- } \\
\text { pie }\end{array}$ & 1 & -1 & 0 & 0 & 0 & 0 & 0 & 0 \\
\hline 812 & $\begin{array}{l}\text { Medizinisches Labora- } \\
\text { torium }\end{array}$ & 2 & -1 & -1 & 0 & 1 & 0 & 0 & 0 \\
\hline 817 & $\begin{array}{l}\text { Nicht ärztliche Thera- } \\
\text { pie und Heilkunde }\end{array}$ & 2 & -2 & -1 & 0 & 1 & 0 & 0 & 0 \\
\hline 811 & Arzt- und Praxishilfe & 3 & -2 & -2 & 0 & 0 & 0 & 0 & 0 \\
\hline 814 & $\begin{array}{l}\text { Human- und Zahnme- } \\
\text { dizin }\end{array}$ & 1 & -1 & 0 & 0 & 1 & 0 & 0 & 0 \\
\hline 813 & $\begin{array}{l}\text { Gesundheits- und Kran- } \\
\text { kenpflege, Rettungsd. } \\
\text { u. Geburtshilfe }\end{array}$ & 2 & -2 & -2 & 0 & 0 & 0 & -1 & -1 \\
\hline 821 & Altenpflege & 3 & -3 & -3 & -1 & 0 & 0 & 0 & 0 \\
\hline
\end{tabular}

Quelle: Forschungsdatenzentrum der Statistischen Ämter des Bundes und der Länder, Mikrozensen 1997-2017 und Volkswirtschaftliche Gesamtrechnungen des Statistischen Bundesamts; Beschäftigtenhistorik der Bundesagentur für Arbeit; Berechnung und Darstellung QuBe-Projekt, sechste Welle; nur Berufsgruppen mit mindestens 20.000 Erwerbstätigen

den. Zum anderen die berufliche Flexibilität der Erwerbspersonen. So reagieren die Erwerbspersonen auf die schlechteren Erwerbschancen in ihren erlernten Berufen und bieten ihre Arbeitskraft stattdessen in anderen, tätigkeitsähnlichen Berufen an.

Tab. 8 im Anhang macht deutlich, dass das Arbeitsangebot vor allem in Berufsgruppen, welche ein mittleres Qualifikationsniveau voraussetzen und vorwiegend von Frauen gewählt werden, in der kurzen Frist zurückgehen könnte. Dies ist ein Ergebnis der langfristigen Trendschätzungen, wo sich ein signifikant positiver Zusammenhang zwischen der Frauenerwerbsquote und der Konjunktur (BIP/Kopf) offenbarte. In den Berufsgruppen „Reinigung“, „Gastronomie“ und „Hotellerie“ zeigt sich verbunden mit berufsspezifischen Abwanderungen sogar langfristig ein geringeres Arbeitsangebot. Von der Krise profitieren können die vorwiegend männlich dominierten Bauberufe. Im Rahmen ihrer beruflichen Mobilität bieten hier Erwerbspersonen aus der Industrie, welche im Zuge der COVID-19-Krise Arbeitsplätze abbaut, ihre Arbeitskraft verstärkt an.

Tab. 7 zeigt mit der Gegenüberstellung der „Erwerbslosen“ nach Berufsgruppen zwischen beiden Szenarien (Basisprojektion und kontrafaktisches Szenario) das Ergebnis der bedarfs- und angebotsseitigen Anpassungsprozesse. Bei der Zahl der Erwerbslosen handelt es sich um die Differenz zwischen den Erwerbspersonen, die ihre Arbeitskraft in einer Berufsgruppe anbieten und dem Bedarf an Erwerbstätigen in dieser Berufsgruppe. Dabei bleibt unberücksichtigt, dass die Erwerbspersonen ihre Arbeitskraft in der Praxis auch in mehreren Berufen anbieten können.

Unter Berücksichtigung der Auswirkungen der COVID-19-Pandemie nimmt die Zahl der Erwerbslosen (in Tausend Personen) vor allem in der „Gastronomie“, der 
Tab. 7 Berufsgruppen mit den 20 stärksten Zuwächsen und zehn stärksten Rückgängen an ,Erwerbslosen" aufgrund der COVID-19-Pandemie (Basisprojektion - kontrafaktisches Szenario) 2020 in Tausend Personen und Anpassung bis 2040

\begin{tabular}{|c|c|c|c|c|c|c|c|c|c|}
\hline \multicolumn{2}{|c|}{ Berufsgruppe } & \multicolumn{8}{|l|}{ Jahr } \\
\hline & & 2020 & 2021 & 2022 & 2023 & 2025 & 2030 & 2035 & 2040 \\
\hline 633 & Gastronomie & 130 & 94 & 3 & -44 & -23 & -15 & -6 & -4 \\
\hline 293 & Speisenzubereitung & 69 & 46 & -2 & -25 & -11 & -8 & -4 & -3 \\
\hline 513 & $\begin{array}{l}\text { Lagerwirtschaft, Post } \\
\text { und Zustellung, Güter- } \\
\text { umschlag }\end{array}$ & 28 & 23 & 19 & 4 & 0 & -5 & -3 & -4 \\
\hline 632 & Hotellerie & 28 & 23 & 5 & -6 & -3 & -6 & -5 & -5 \\
\hline 251 & $\begin{array}{l}\text { Maschinenbau- und } \\
\text { Betriebstechnik }\end{array}$ & 18 & 13 & 4 & -3 & -1 & -3 & -2 & -2 \\
\hline 521 & $\begin{array}{l}\text { Fahrzeugführung im } \\
\text { Straßenverkehr }\end{array}$ & 18 & 28 & 18 & -1 & -4 & -3 & -2 & -3 \\
\hline 721 & $\begin{array}{l}\text { Versicherungs- und } \\
\text { Finanzdienstleistungen }\end{array}$ & 16 & 10 & 10 & 2 & 1 & 2 & 4 & 3 \\
\hline 242 & Metallbearbeitung & 14 & 9 & 3 & -1 & 0 & -1 & -1 & -2 \\
\hline 621 & $\begin{array}{l}\text { Verkauf (ohne Produkt- } \\
\text { spezialisierung) }\end{array}$ & 14 & 14 & 12 & -1 & -5 & -5 & -2 & 1 \\
\hline 713 & $\begin{array}{l}\text { Unternehmensorganisation } \\
\text { und -strategie }\end{array}$ & 13 & 7 & 9 & -2 & -3 & -2 & 1 & 0 \\
\hline 631 & Tourismus und Sport & 12 & 11 & 3 & -1 & -1 & -1 & -1 & 0 \\
\hline 623 & $\begin{array}{l}\text { Verkauf von Lebensmit- } \\
\text { teln }\end{array}$ & 11 & 8 & 2 & -2 & -1 & -2 & -1 & 0 \\
\hline 292 & $\begin{array}{l}\text { Lebensmittel- und Ge- } \\
\text { nussmittelherstellung }\end{array}$ & 10 & 7 & 1 & -2 & -1 & -1 & -1 & 0 \\
\hline 711 & $\begin{array}{l}\text { Geschäftsführung und } \\
\text { Vorstand }\end{array}$ & 10 & 8 & 5 & -1 & -1 & -2 & -1 & -1 \\
\hline 531 & $\begin{array}{l}\text { Objekt-, Personen-, } \\
\text { Brandschutz, Arbeitssi- } \\
\text { cherheit }\end{array}$ & 10 & 12 & 9 & 2 & 0 & -3 & -3 & -4 \\
\hline 252 & $\begin{array}{l}\text { Fahrzeug-, Luft-, } \\
\text { Raumfahrt- und Schiff- } \\
\text { bautechnik }\end{array}$ & 8 & 3 & 2 & -1 & -1 & -1 & 0 & 0 \\
\hline 273 & $\begin{array}{l}\text { Technische Produkti- } \\
\text { onsplanung und -steue- } \\
\text { rung }\end{array}$ & 8 & 6 & 1 & -2 & -1 & -2 & -1 & -1 \\
\hline 611 & Einkauf und Vertrieb & 7 & 6 & 3 & -2 & -3 & -3 & -2 & -2 \\
\hline 244 & $\begin{array}{l}\text { Metallbau und } \\
\text { Schweißtechnik }\end{array}$ & 7 & 5 & 2 & 0 & 0 & -1 & -1 & -1 \\
\hline 844 & $\begin{array}{l}\text { Lehrtätigkeit an außer- } \\
\text { schulischen Bildungs- } \\
\text { einrichtungen }\end{array}$ & 7 & 0 & -1 & -2 & -1 & 0 & 0 & 0 \\
\hline \multicolumn{10}{|l|}{$\cdots$} \\
\hline 843 & $\begin{array}{l}\text { Lehr- und Forschungs- } \\
\text { tätigkeit an Hochschu- } \\
\text { len }\end{array}$ & -4 & -7 & -12 & -17 & -20 & -17 & -17 & -15 \\
\hline 111 & Landwirtschaft & -6 & -6 & -2 & 0 & 1 & -1 & -1 & 0 \\
\hline
\end{tabular}


Tab. 7 (Fortsetzung)

\begin{tabular}{|c|c|c|c|c|c|c|c|c|c|}
\hline \multicolumn{2}{|c|}{ Berufsgruppe } & \\
\hline & & 2020 & 2021 & 2022 & 2023 & 2025 & 2030 & 2035 & 2040 \\
\hline 541 & Reinigung & -7 & 20 & 33 & 17 & 1 & -9 & -7 & -5 \\
\hline 817 & $\begin{array}{l}\text { Nicht ärztliche Thera- } \\
\text { pie und Heilkunde }\end{array}$ & -10 & -8 & -8 & -7 & -2 & -1 & -2 & -1 \\
\hline 814 & $\begin{array}{l}\text { Human- und Zahnmedi- } \\
\text { zin }\end{array}$ & -11 & -11 & -13 & -13 & -6 & -4 & -4 & -3 \\
\hline 832 & $\begin{array}{l}\text { Hauswirtschaft und } \\
\text { Verbraucherberatung }\end{array}$ & -11 & -8 & 0 & 2 & 4 & -1 & -2 & -2 \\
\hline 831 & $\begin{array}{l}\text { Erziehung, Sozialarbeit, } \\
\text { Heilerziehungspflege }\end{array}$ & -11 & -10 & -2 & 1 & 4 & -1 & 0 & 2 \\
\hline 811 & Arzt- und Praxishilfe & -25 & -17 & -13 & -9 & 2 & 1 & 0 & 1 \\
\hline 821 & Altenpflege & -44 & 2 & 7 & 6 & 4 & 3 & 2 & 2 \\
\hline 813 & $\begin{array}{l}\text { Gesundheits- und Kran- } \\
\text { kenpflege, Rettungs- } \\
\text { dienst und Geburtshilfe }\end{array}$ & -46 & -20 & -16 & -13 & 0 & 4 & 8 & 14 \\
\hline
\end{tabular}

Quelle: Forschungsdatenzentrum der Statistischen Ämter des Bundes und der Länder, Mikrozensen 1997-2017 und Volkswirtschaftliche Gesamtrechnungen des Statistischen Bundesamts; Beschäftigtenhistorik der Bundesagentur für Arbeit; Berechnung und Darstellung QuBe-Projekt, sechste Welle; nur Berufsgruppen mit mindestens 20.000 Erwerbstätigen

„Speisenzubereitung“, „Lagerwirtschaft, Post und Zustellung, Güterumschlag“ und „Hotellerie“ zu. Ein langfristig höheres Arbeitsangebot als Arbeitskräftebedarf zeigt sich jedoch nur in den ,Versicherungs- und Finanzdienstleistungen“. In allen anderen Berufsgruppen, deren Erwerbslosenzahlen im Jahr 2020 ansteigen, ist langfristig sogar ein Rückgang im Vergleich zum kontrafaktischen Szenario erkennbar, weil auch das Arbeitsangebot leicht abnimmt. Damit trifft die Krise in der kurzen und mittleren Frist vor allem Berufe, in welchen Fachkräfte aus dem mittleren Qualifikationsbereich, aber auch Un- und Angelernte, eine Erwerbstätigkeit ausüben.

Zu den Berufsgruppen mit einer geringeren Anzahl an Erwerbslosen im Jahr 2020 zählen zum einen die Berufe des Gesundheitswesens mit einem zunehmenden Bedarf an Erwerbstätigen, aber auch die Berufsgruppen ,Hauswirtschaft- und Verbraucherberatung“ oder „Reinigung“, bei denen sich ein kleiner Teil der Erwerbspersonen vom Arbeitsmarkt zurückzieht. Langfristig ergibt sich aus QINFORGE, dass in der „Altenpflege“ und in den Bereichen „Gesundheits- und Krankenpflege, Rettungsdienst und Geburtshilfe" etwas mehr Erwerbspersonen ihre Arbeitskraft anbieten könnten, obwohl auch der Bedarf an Erwerbstätigen in der Basisprojektion geringfügig höher ausfällt als im kontrafaktischen Szenario. Dies ist darauf zurückzuführen, dass sich die Erwerbschancen in den anderen Berufen, welche qualifizierte Pflegekräfte ausüben können, in der Basisprojektion im Vergleich zu kontrafaktischen Szenario geringer sind. Ein ähnlicher Mechanismus zeigt sich in ,Lehr- und Forschungstätigkeiten an Hochschulen“. Hier ist der Bedarf an Erwerbstätigen in der „Basisprojektion“ höher, die Erwerbs- und Entlohnungschancen in anderen Berufen aber weiterhin besser, wodurch die Differenz der benötigten Erwerbstätigen und potenziell zur Verfügung stehenden Erwerbspersonen im Beruf in der Basisprojektion geringer ausfällt. 


\section{Fazit}

Die Qualifikations- und Berufsprojektionen haben zum Ziel, mittel- und langfristige berufliche Passungsprobleme aufzuzeigen, um für die Arbeits- und Bildungspolitik entsprechende Handlungsfelder benennen zu können. Dieser Bericht stellt das für die Projektionen verwendete Modellsystem QINFORGE vor. Das Alleinstellungsmerkmal von QINFORGE liegt in der empirisch fundierten Modellierung beruflicher Anpassungsprozesse durch die Verwendung beruflicher Mobilitätsmatrizen (Abschn. 2). So nimmt die berufsspezifische Entlohnung Einfluss auf die nachgefragte Anzahl an Gütern und Dienstleistungen - wie auch das zur Verfügung stehende Arbeitsangebot in einem Beruf. Ebenso werden Erwerbsquoten in Abhängigkeit von der wirtschaftlichen Entwicklung modelliert.

Die wirtschaftlichen Einschnitte in Folge der COVID-19-Pandemie bieten einen Rahmen, um die Wirkung der endogen modellierten Anpassungsprozesse zu demonstrieren und entsprechende Konsequenzen der COVID-19-Pandemie auf die berufsspezifischen Arbeitsmärkte in der kurzen, mittleren und langen Frist zu verdeutlichen. Hierfür werden zwei Szenarien miteinander verglichen. Ein kontrafaktisches Szenario schreibt langfristige Verhaltensweisen und Trends ausgehend vom Jahr 2019 fort. Die Basisprojektion versucht hingegen, bereits die Konjunktur des Jahres 2020 mit abzubilden, indem eine geringere Investitionsneigung, ein zurückgehender Konsum, ein geringerer Außenhandel und eine Reduzierung der Arbeitszeiten berücksichtigt wird. Ebenso wird das am 3. Juni 2020 verabschiedete Konjunkturpaket in Höhe von 130 Mrd. $€$ berücksichtigt (siehe Abschn. 3).

Die COVID-19-Pandemie hat Deutschland in einer robusten wirtschaftlichen Lage getroffen. Die makroökonomischen Fundamentalvariablen der Wirtschaft waren vor dem Wirtschaftseinbruch gesund und der Arbeitsmarkt stabil - mit einer Erwerbslosenquote auf historisch niedrigem Niveau. Die Ergebnisse des Szenario-Vergleichs offenbaren, dass die Krise die Wirtschaftsleistung Deutschlands langfristig um drei Jahre zurückwerfen wird. Trotz der möglichen Kurzarbeit wird aufgrund der Corona-Pandemie auch die Erwerbslosigkeit ansteigen. Für die Jahre 2020 und 2021 prognostiziert die Basisprojektion jeweils rund 600.000 Erwerbstätige weniger als im kontrafaktischen Szenario. Da sich aufgrund der schlechteren Arbeitsmarktlage aber auch einige Erwerbspersonen vom Arbeitsmarkt zurückziehen werden, steigt die Zahl der Erwerbslosen in beiden Jahren jedoch nur um rund 400.000 Personen im Vergleich zum kontrafaktischen Szenario an.

Die Unterbeschäftigung muss in der Veränderung der Jahresarbeitszeit berücksichtigt werden und beeinflusst dadurch auch die Entwicklung des Stundenlohns: So hemmt die Corona-Pandemie vor allem in der kurzen und mittleren Frist das Lohnwachstum, welches in einem durch Fachkräfteengpässe gekennzeichneten Arbeitsmarkt im kontrafaktischen Szenario möglich gewesen wäre. Dies führt dazu, dass die Basisprojektion zum Projektionsende im Jahr 2040 in etwa genauso viele Personen in Erwerbstätigkeit berechnet wie im kontrafaktischen Szenario.

Die Annahmen der Branchenentwicklungen, welche sich auf aktuelle Erhebungen und Registerdaten mit Datenstand September 2020 stützen, offenbaren, dass das Arbeitskräfteangebot und der -bedarf nach Berufen durch die Krise unterschiedlich betroffen sein wird. Da die „Shutdowns“ vorwiegend den Tourismus, die Gastrono- 
mie sowie Kunst und Kultur treffen, zeigt sich in den entsprechenden Berufen der stärkste Rückgang im Erwerbstätigenbedarf und in den Entlohnungen. So wird es in der Basisprojektion rund fünf Jahre dauern, bis eine Erwerbstätigkeit und ein nominales Lohnniveau erreicht wird, welches der Berechnung im kontrafaktischen Szenario entspricht. Bei den Erwerbsquoten wird deutlich, dass sich vor allem Frauen im mittleren Qualifikationsbereich durch die Krise vom Arbeitsmarkt zurückziehen und damit auf Einkommen verzichten.

In Berufen des Gesundheitsbereichs steigt hingegen in der kurzen Frist die Nachfrage nach Erwerbstätigen. Aufgrund der Budgetrestriktionen des Gesundheitsbereichs werden die ebenfalls kurzfristigen Lohnsteigerungen von QINFORGE modellendogen als Vorzieheffekte gedeutet. Langfristig zeigt sich in der Basisprojektion im Gesundheitsbereich - und hier vor allem bei den Pflegeberufen - ein leicht höheres Arbeitsangebot als im kontrafaktischen Szenario. Dies ist jedoch weniger auf einen Pull-Faktor der Pflege in Form einer höheren Entlohnung, sondern eher auf geringere Pull-Faktoren aus anderen Berufen zurückzuführen. Durch die COVID19-Pandemie wird die Entlohnung in alternativen Berufen, die für Pflegefachkräfte in Frage kommen, geringer. Eine ähnliche Arbeitsmarktsituation ergibt sich auch in den Bauberufen: $\mathrm{Da}$ in der Basisprojektion in der Industrie mehr Arbeitsplätze verloren gehen als im kontrafaktischen Szenario, bieten die vorwiegend männlichen Erwerbspersonen ihre Arbeitskraft eher in den tätigkeitsähnlichen Bauberufen an. Damit deutet sich an, dass die COVID-19-Krise das Arbeitsangebot in Berufen erhöhen könnte, in denen bereits heute ein Fachkräfteengpass diagnostiziert wird (Statistik der BA 2019), die aber bislang für die Erwerbspersonen weniger attraktiv waren. Gleichwohl stellt das knappe Arbeitsangebot langfristig einen hemmenden Faktor für das Wirtschaftswachstum dar. Auch in der Basisprojektion wird - insbesondere in den Pflegeberufen - das Arbeitsangebot langfristig nicht ausreichen, um die Arbeitsnachfrage zu befriedigen. Neben entsprechenden Qualifizierungsmaßnahmen wird deshalb auch Zuwanderung notwendig sein, um die Zahl der Erwerbspersonen zu erhöhen.

Für die Analyse wurde die COVID-19-Krise als externer Schock betrachtet. Sie zeigt, dass vor allem Personen in Berufen, die ein mittleres Qualifikationsniveau voraussetzen, in den Entlohnungs- und Erwerbschancen in der kurzen und mittleren Frist beeinträchtigt werden. Hier könnten individuelle Qualifizierungsmaßnahmen helfen, um die Beschäftigung in weniger von der Krise betroffenen Berufen zu erhöhen. Langfristig können sie jedoch wieder im gleichen Maße am Arbeitsmarkt teilhaben wie in einem kontrafaktischen Szenario - sofern die Arbeitswelt wieder in die alten Verhaltensmuster zurückfällt. Dies muss aber nicht der Fall sein. So zeigt sich in der Krise eine weitaus stärkere Nutzung von Homeoffice und Videokonferenztools, welche auch nach der ökonomischen Bewältigung der Krise fortbestehen kann. Hier ist bekannt, dass gerade die Zugänge zu Homeoffice-Tätigkeiten nach Qualifikationsniveau ungleich verteilt sind (Mergener 2020). Die Auswirkungen einer solchen veränderten Arbeitsweise auf die beruflichen Beschäftigungs- und Entlohnungschancen müssten in weiteren Szenarien untersucht werden. 


\section{Anhang}

Tab. 8 Berufsgruppen mit den 20 stärksten Rückgängen und zehn stärksten Zuwächsen an Erwerbspersonen aufgrund der COVID-19-Pandemie (Basisprojektion - kontrafaktisches Szenario) 2020 in Prozent und Anpassung bis 2040

\begin{tabular}{|c|c|c|c|c|c|c|c|c|c|}
\hline & \multicolumn{8}{|l|}{ Jahr } \\
\hline \multicolumn{2}{|c|}{ Berufsgruppe } & \multirow{2}{*}{$\frac{2020}{-2}$} & \multirow{2}{*}{$\frac{2021}{-1}$} & \multirow{2}{*}{$\frac{2022}{1}$} & \multirow{2}{*}{$\frac{2023}{1}$} & \multirow{2}{*}{$\frac{2025}{1}$} & \multirow{2}{*}{$\frac{2030}{0}$} & \multirow{2}{*}{$\frac{2035}{0}$} & \multirow{2}{*}{$\frac{2040}{0}$} \\
\hline 832 & $\begin{array}{l}\text { Hauswirtschaft und } \\
\text { Verbraucherberatung }\end{array}$ & & & & & & & & \\
\hline 541 & Reinigung & -1 & -1 & 0 & 0 & -1 & -1 & -1 & 0 \\
\hline 624 & $\begin{array}{l}\text { Verkauf von drogerie- } \\
\text { und apothekenüblichen } \\
\text { Waren, Sanitäts- und } \\
\text { Medizinbedarf }\end{array}$ & -1 & -1 & 0 & 0 & 0 & 0 & 0 & 0 \\
\hline 623 & $\begin{array}{l}\text { Verkauf von Lebensmit- } \\
\text { teln }\end{array}$ & -1 & -1 & -1 & 0 & 0 & 0 & 0 & 0 \\
\hline 282 & Textilverarbeitung & -1 & -1 & 0 & -1 & -1 & -2 & -2 & -1 \\
\hline 811 & Arzt- und Praxishilfe & -1 & 0 & 1 & 1 & 1 & 0 & 0 & 1 \\
\hline 821 & Altenpflege & -1 & 0 & 1 & 1 & 1 & 1 & 1 & 1 \\
\hline 714 & Büro und Sekretariat & -1 & -1 & 0 & 0 & 0 & 0 & 0 & 0 \\
\hline 122 & Floristik & -1 & -1 & 0 & 0 & 0 & 0 & 0 & 0 \\
\hline 115 & Tierpflege & -1 & -1 & 0 & 0 & 0 & -1 & -1 & 0 \\
\hline 621 & $\begin{array}{l}\text { Verkauf (ohne Produkts- } \\
\text { pezialisierung) }\end{array}$ & -1 & -1 & 0 & 0 & 0 & 0 & 0 & 0 \\
\hline 633 & Gastronomie & -1 & -3 & -5 & -5 & -4 & -2 & -1 & 0 \\
\hline 632 & Hotellerie & -1 & -2 & -3 & -3 & -3 & -2 & -2 & -2 \\
\hline 733 & $\begin{array}{l}\text { Medien-, Dokumentati- } \\
\text { ons- und Informationsd. }\end{array}$ & -1 & -1 & 0 & 0 & 0 & 0 & 0 & 0 \\
\hline 625 & $\begin{array}{l}\text { Buch-, Kunst-, Antiqui- } \\
\text { täten- und Musikfach- } \\
\text { handel }\end{array}$ & -1 & -1 & 0 & 0 & 0 & 0 & 0 & 0 \\
\hline 823 & Körperpflege & -1 & 0 & 0 & 0 & -1 & -1 & -1 & -1 \\
\hline 732 & Verwaltung & -1 & -1 & 0 & 0 & 0 & 0 & 0 & 0 \\
\hline 622 & $\begin{array}{l}\text { Verkauf von Bekleidung, } \\
\text { Elektronik, Kraftfahr- } \\
\text { zeugen und Hartwaren }\end{array}$ & -1 & -1 & 0 & 0 & 0 & 0 & 0 & 0 \\
\hline 112 & Tierwirtschaft & -1 & -1 & 0 & 1 & 0 & 0 & 0 & 1 \\
\hline 812 & $\begin{array}{l}\text { Medizinisches Laborato- } \\
\text { rium }\end{array}$ & -1 & -1 & 0 & 0 & 0 & 0 & 0 & 0 \\
\hline$\ldots$ & & & & & & & & & \\
\hline 262 & Energietechnik & 0 & 0 & 0 & 0 & 0 & 0 & 0 & 0 \\
\hline 223 & $\begin{array}{l}\text { Holzbe- und -verarbei- } \\
\text { tung }\end{array}$ & 0 & 0 & 0 & 0 & 0 & 0 & 0 & 0 \\
\hline 211 & $\begin{array}{l}\text { Berg-, Tagebau und } \\
\text { Sprengtechnik }\end{array}$ & 0 & 0 & 0 & 0 & -1 & 0 & 0 & 0 \\
\hline 342 & $\begin{array}{l}\text { Klempnerei, Sanitär-, } \\
\text { Heizungs- und Klima- } \\
\text { technik }\end{array}$ & 0 & 0 & 0 & 0 & 0 & -1 & -1 & 0 \\
\hline 322 & Tiefbau & 0 & 0 & 1 & 0 & 0 & 0 & 0 & 0 \\
\hline
\end{tabular}


Tab. 8 (Fortsetzung)

\begin{tabular}{|c|c|c|c|c|c|c|c|c|c|}
\hline \multicolumn{2}{|c|}{ Berufsgruppe } & \multicolumn{8}{|l|}{ Jahr } \\
\hline & & 2020 & 2021 & 2022 & 2023 & 2025 & 2030 & 2035 & 2040 \\
\hline 212 & $\begin{array}{l}\text { Naturstein- und Mi- } \\
\text { neralaufbereitung und } \\
\text {-verarbeitung und Bau- } \\
\text { stoffherstellung }\end{array}$ & 0 & 1 & 1 & 0 & 0 & 0 & 0 & 0 \\
\hline 332 & $\begin{array}{l}\text { Maler- und Lackierer-, } \\
\text { Stuckateurarbeiten, } \\
\text { Bauwerksabdichtung, } \\
\text { Holz- und Bautenschutz }\end{array}$ & 0 & 1 & 1 & 0 & 0 & -1 & -1 & 0 \\
\hline 333 & $\begin{array}{l}\text { Aus- und Trockenbau, } \\
\text { Isolierung, Zimmerei, } \\
\text { Glaserei, Rollladen- und } \\
\text { Jalousiebau }\end{array}$ & 0 & 1 & 1 & 0 & 0 & -1 & -1 & 0 \\
\hline 331 & Bodenverlegung & 0 & 1 & 1 & 0 & 0 & -1 & -1 & 0 \\
\hline 321 & Hochbau & 0 & 1 & 1 & 1 & 0 & -1 & -1 & 0 \\
\hline
\end{tabular}

Quelle: Forschungsdatenzentrum der Statistischen Ämter des Bundes und der Länder, Mikrozensen 1997-2017 und Volkswirtschaftliche Gesamtrechnungen des Statistischen Bundesamts; Beschäftigtenhistorik der Bundesagentur für Arbeit; Berechnung und Darstellungen QuBe-Projekt, sechste Welle; nur Berufsgruppen mit mindestens 20.000 Erwerbstätigen.

Funding Open Access funding enabled and organized by Projekt DEAL.

Open Access Dieser Artikel wird unter der Creative Commons Namensnennung 4.0 International Lizenz veröffentlicht, welche die Nutzung, Vervielfältigung, Bearbeitung, Verbreitung und Wiedergabe in jeglichem Medium und Format erlaubt, sofern Sie den/die ursprünglichen Autor(en) und die Quelle ordnungsgemäß nennen, einen Link zur Creative Commons Lizenz beifügen und angeben, ob Änderungen vorgenommen wurden.

Die in diesem Artikel enthaltenen Bilder und sonstiges Drittmaterial unterliegen ebenfalls der genannten Creative Commons Lizenz, sofern sich aus der Abbildungslegende nichts anderes ergibt. Sofern das betreffende Material nicht unter der genannten Creative Commons Lizenz steht und die betreffende Handlung nicht nach gesetzlichen Vorschriften erlaubt ist, ist für die oben aufgeführten Weiterverwendungen des Materials die Einwilligung des jeweiligen Rechteinhabers einzuholen.

Weitere Details zur Lizenz entnehmen Sie bitte der Lizenzinformation auf http://creativecommons.org/ licenses/by/4.0/deed.de.

\section{Literatur}

Ahlert G, Distelkamp M, Lutz C, Meyer B, Mönnig A, Wolter MI (2009) Das IAB/INFORGE-Modell. In: Schnur P, Zika G (Hrsg) Das IAB/INFORGE-Modell. Ein sektorales makroökonometrisches Projektions- und Simulationsmodell zur Vorausschätzung des längerfristigen Arbeitskräftebedarfs. IABBibliothek. Bertelsmann, Bielefeld

Aigner-Walder B, Döring T (2014) The effects of population ageing on private consumption — a simulation for Austria based on household data up to 2050. Eur Econ Rev 2:63-80. https://doi.org/10.14208/ BF03353833

Bachem A, Korte B (1979) On the RAS algorithm. Computing 23:189-198

Bakens J, Fouarge D, Peeters T (2018) Labour market forecasts by education and occupation up to 2022. ROA Technical Reports No. 003. Maastricht University, Maastricht 
Bonin H, Schneider M, Quinke H, Arens T (2007) Zukunft von Bildung und Arbeit. Perspektiven von Arbeitskräftebedarf und -angebot bis 2020. IZA Research Report 9

Bundesagentur für Arbeit (2019) Berichte: Blickpunkt Arbeitsmarkt - Fachkräfteengpassanalyse. Dezember 2019. Bundesagentur für Arbeit Statistik/Arbeitsmarktberichterstattung, Nürnberg

Bundesamt für Statistik (2019) Bildungsperspektiven. Szenarien 2018-2027 für das Bildungssystem. Bundesamt für Statistik, Neuchâtel

Bundesministerium für Wirtschaft und Energie (2021) Jahreswirtschaftsbericht 2021. Corona-Krise überwinden, wirtschaftliche Erholung unterstützen, Strukturen stärken. Bundesministerium für Wirtschaft und Energie, Berlin

Bureau of Labor Statistics (2018) Employment projections methodology. https://www.bls.gov/emp/ documentation/projections-methods.htm. Zugegriffen: 23. Juli 2019

Büttner T, Rässler S (2008) Multiple imputation of right-censored wages in the German IAB employment sample considering heteroscedasticity. IAB-Discussion Paper 44/2008

Deutsche Bundesbank (2017) Demographic change, immigration and the potential output of German economy. Monthly Report April 2017, S 35-47

Deutsche Bundesbank (2020) Perspektiven der deutschen Wirtschaft für die Jahre 2021 bis 2023. Monatsbericht Dezember 2020

Dunn ESJ (1960) A statistical and analytical technique for regional analysis. Papers and Proceedings of the Regional Science Association, Bd. 6, S 98-112

Europäisches Zentrum für die Förderung der Berufsbildung (Cedefop) (2010) Skills supply and demand in Europe. Medium-term forecast up to 2020. Publications Office of the European Union, Luxembourg

Europäisches Zentrum für die Förderung der Berufsbildung (Cedefop) (2018) Skills forecast. Trends and challenges to 2030. Publications Office of the European Union, Luxembourg

France Stratégie, Dares (2018) Prospective des Métiers et Qualifications (PMQ): Bilan et Perspectives. France Stratégie, Paris

Fuchs J, Söhnlein D, Weber B, Weber E (2017) Forecasting labour supply and population: an integrated stochastic model. IAB-Discussion Paper 01/2017

Gorodetski K, Mönnig A, Wolter MI (2016) Zuwanderung nach Deutschland. Mittel- und langfristige Projektionen mit dem Modell TINFORGE. GWS Discussion Paper 2016/01. Gesellschaft für Wirtschaftliche Strukturforschung, Osnabrück

Helmrich R, Zika G (2019) Prognosen, Projektionen und Szenarien. In: Baur N, Blasius J (Hrsg) Handbuch Methoden der empirischen Sozialforschung. VS, Wiesbaden, S 231-246

Hutter C, Weber E (2020) Corona-Krise: die transformative Rezession. Wirtschaftsdienst 100:429-431

International Energy Agency (IEA) (2019) World Energy Outlook 2019. IEA, Paris

International Monetary Fund (IMF) (2019) World economic outlook database

Kalinowski M, Mönnig A, Söhnlein D (2021) Annahmen, Modellierung und Ergebnisse der Angebotsprojektion nach Qualifikationsstufen und Berufen bis zum Jahr 2040. Bundesinstitut für Berufsbildung, Bonn

Klauder W (1986) Technischer Fortschritt und Beschäftigung. Mitt Arbeitsmarkt Berufsforsch 19:1-19

Kriechel B, Long K, Millar P, Pollitt H, Vetter T, Vogler-Ludwig K, Wilson R (2013) Arbeitsmarkt 2030. Methodenbericht. W. Bertelsmann, München

Lehr U, Lutz C, Ulrich P (2014) Economic evaluation of climate protection measures in Germany. Int J Energy Econ Policy 4:693-705

Lucas RE (1976) Econometric policy evaluation: a critique. Carnegie Rochester Conf Ser Publ Policy $1: 19-46$

Maier T, Helmrich R (2012) Creating the initial vocational qualification from the German Microcensus. Paper presented at the Eighth International Conference on Social Science Methodology, Sydney

Maier T, Helmrich R, Schandock M, Neuber-Pohl C, Bott P (2014) Löhne und berufliche Flexibilitäten als Determinanten des interaktiven QuBe-Arbeitsmarktmodells. Ein Methodenbericht zur Basisprojektion der 3. Welle der BIBB-IAB Qualifikations- und Berufsfeldprojektionen Wissenschaftliche Diskussionpapiere..

Maier T, Mönnig A, Zika G (2015) Labour demand in Germany by industrial sector, occupational field and qualification until 2025-model calculations using the IAB/INFORGE model. Econ Syst Res 27:19-42

Maier T, Neuber-Pohl C, Mönnig A, Zika G, Kalinowski M (2017) Modelling reallocation processes in long-term labour market projections. J Labour Mark Res 50:67-90

Maier T, Zika G, Kalinowski M, Steeg S, Mönnig A, Wolter MI, Hummel M, Schneemann C (2020) COVID-19-Krise: Die Arbeit geht weiter, der Wohlstand macht Pause. BIBB-Report 4/2020. Bundesinstitut für Berufsbildung, Bonn 
Meagher GA, Pang F, Wilson R (2015) Interfacing a CGE labour market model with the E3ME multisector macroeconomic model. Melbourne. Centre of Policy Studies Working Paper

Mergener A (2020) Berufliche Zugänge zum Homeoffice. Ein tätigkeitsbasierter Ansatz zur Erklärung von Chancenungleichheit beim Homeofficezugang. Kolner Z Soz Sozpsychol 72:511-534

Meyer B, Bockermann A, Ewerhart G, Lutz C (1999) Marktkonforme Umweltpolitik. Wirkungen auf Luftschadstoffemissionen, Wachstum und Struktur der Wirtschaft. Umwelt und Ökonomie. Springer, Berlin, Heidelberg

Mönnig A (2016) The European Monetary Union break-up: an economic experiment on the return of the deutsche mark. Econ Syst Res 28:497-517

Mönnig A, Wolter MI (2020) TINFORGE - Trade in INFORGE. Methoden-Update 2020. Osnabrück. GWS Discussion Paper 2020/4

Mönnig A, Maier T, Zika G (2019) Economy 4.0_digitalisation and its effect on wage inequality. J Econ Stat 329:363-398

Mönnig A, Schneemann C, Weber E, Zika G, Helmrich R (2018) Elektromobilität 2035 - Effekte auf Wirtschaft und Erwerbstätigkeit durch die Elektrifizierung des Antriebsstrangs von Personenkraftwagen. Nürnberg. IAB-Forschungsbericht 8/2018

Mönnig A, Schneemann C, Weber E, Zika G (2020) Das Klimaschutzprogramm 2030. Effekte auf Wirtschaft und Erwerbstätigkeit durch das Klimaschutzprogramm 2030 der Bundesregierung. Nürnberg. IAB-Discussion Paper 02/2020

Organisation for Economic Co-operation and Development (OECD) (2020) OECD-Wirtschaftsausblick, Ausgabe 2020/2 (Kurzfassung). OECD Publishing, Paris https://doi.org/10.1787/e0461d96-de

Organisation for Economic Co-operation and Development (OECD), Food and Agriculture Organization (FAO) (2019) OECD-FAO agricultural outlook 2019-2028. OECD Publishing, Paris

Peichl A, Schneider H, Siegloch S (2010) Documentation IZAMOD: the IZA policy simulation MOdel. IZA Discussion Paper No. 4865. Forschungsinstitut zur Zukunft der Arbeit, Bonn

Pollitt H, Lewney R, Mercure J-F (2019) Conceptual differences between macro-econometric and CGE models Paper presented at the 2th International Input-Output Association Conference. Glasgow

Prognos (2013) Das Prognos Weltwirtschaftsmodell VIEW. Prognos Welt Report 2013

Richter J (1991) Aktualisierung und Prognose technischer Koeffizienten in gesamtwirtschaftlichen InputOutput Modellen. Springer, Heidelberg

Sarno L, Science M, Taylor P (2002) Purchasing power parity and the real exchange rate. IMF Staff Papers 49, S 65-105

Sonnenburg A, Stöver B, Ulrich P, Wolter MI (2015) Auswirkungen des demographischen Wandels auf Branchen in Deutschland und potenzielle Rückwirkungen auf Hamburg - Endbericht. Hamburg. Studie im Auftrag der Freien und Hansestadt Hamburg, Behörde für Wirtschaft, Verkehr und Innovation,

Statistics Sweden (2017) Trender och Prognoser 2017 - befolkningen - utbildningen - arbetsmarknaden med sikte på år 2035. Statistics Sweden,

Statistisches Bundesamt (StBA) (2008) Klassifikation der Wirtschaftszweige. Mit Erläuterungen. Statistisches Bundesamt, Wiesbaden

Statistisches Bundesamt (StBA) (2020um) Bruttoinlandsprodukt im 3. Quartal 2020 um 8,2\% höher als im Vorquartal. Pressemitteilung Nr. 432 vom 30. Oktober 2020. StBA, Wiesbaden

Stöver B, Wolter MI (2015) Demographic change and consumption-how ageing affects the level and structure of private consumption. In: Meade DS (Hrsg) Quest of the craft-economic modeling for the 21st century. Firenze University Press, Firenze, S 195-209

Vereinigung der Bayrischen Wirtschaft e. V. (vbw) (2019) Arbeitslandschaft 2025. vbw, München

West GR (2006) Comparison of input-output, input-output + econometric and computable general equilibrium impact models at the regional level. Econ Syst Res 7:209-227

Wilson R, Lindley R (2007) Pan-European skills forecasts. In: Zukersteinova A, Strietska-Ilina O (Hrsg) Towards European skill needs forecasting. Cedefop Panorama series, Bd. 137. Office for Official Publications of the European Communities, S 7-26

Wilson R, May-Gillings M, Pirie J, Beaven R (2016) Working futures 2014-2024: technical report on sources and methods

Wolter MI, Zika G, Maier T (2013) Future skilled-labour markets in Germany: from model-based calculations to scenarios. Statistika 93:70-90

Wolter MI, Helmrich R, Schneemann C, Weber E, Zika G (2020) Auswirkungen des Corona-Konjunkturprogramms auf Wirtschaft und Erwerbstätigkeit. Nürnberg. IAB-Discussion Paper 18/2020 
Zika G, Maier T, Mönnig A (2017) Auswirkungen der Zuwanderung Geflüchteter auf Wirtschaft und Arbeitsmarkt. Berechnungen mit den BIBB-IAB-Qualifikations- und Berufsfeldprojektionen Wissenschaftliche Diskussionspapiere des BIBB, Heft 184. Bundesinstitut für Berufsbildung, Bonn

Zika G, Schneemann C, Grossmann A, Kalinowski M, Maier T, Mönnig A, Parton F, Winnige S, Wolter MI (2019) BMAS-Prognose „Digitalisierte Arbeitswelt“. IAB-Forschungsbericht 5/2019. Institut für Arbeitsmarkt- und Berufsforschung, Nürnberg

Hinweis des Verlags Der Verlag bleibt in Hinblick auf geografische Zuordnungen und Gebietsbezeichnungen in veröffentlichten Karten und Institutsadressen neutral. 